\title{
Argument Contained Ellipsis
}

\author{
Christopher Kennedy \\ University of California, Santa Cruz \\ May 1994
}

1 Introduction 0

Wasow (1972) notes the ungrammaticality of verb phrase ellipsis in sentences such as (1):

*A proof that God exists does.

Wasow accounts for (1) and similar sentences with a constraint that prohibits an elliptical relation from holding between two VPs when one is contained in the subject of the other. Jorge Hankamer (personal communication) points out that this constraint is too strong, however. Configurations of the type specified by Wasow are grammatical just in case the subjects of the elliptically related VPs are identical:

(2a) Every man who said he would buy some salmon did.

(2b) *Every man who said George would buy some salmon did.

In fact, the issue is even more general than Hankamer's examples indicate: in any structure in which one of two elliptically related VPs is embedded in an argument of the other, the arguments must be identical. In (3a-b), which involve Antecedent Contained Deletion (ACD), the internal arguments of the elliptically related VPs must be identical.

(3a) Polly visited every town Eric did.

(3b) *Polly visited every town in every country Eric did.

(3a) has a single interpretation in which for every town Eric visited, Polly visited it too. An interpretation of (3b) "for every country that Eric visited, Polly visited all of its towns" is strongly ungrammatical. The crucial difference between (3a) and (3b) is that in (3a), the direct objects of both the overtly expressed and elided verbs are understood to be the same, whereas in $(3 \mathrm{~b})$ they differ. If ellipsis has not occurred, a sentence with the meaning of (3b) is perfectly grammatical:

(3c) Polly visited every town in every country Eric visited.

(4a-c) indicate that similar facts hold of indirect objects.

(4a) Eric sent letters to every senator Polly did.

(4b) *Eric sent letters to every aide of every senator Polly did.

(4c) Eric sent letters to every aide of every senator Polly sent letters to.

\footnotetext{
${ }^{0}$ I would like to thank Mary Dalrymple, Jorge Hankamer, Jim McCloskey, and Kari Swingle for stimulating discussion of the ideas explored in this paper and for comments on earlier drafts. Thanks also to Bill Ladusaw, whose observations clarified my thinking on several important issues. I am particularly grateful to Sandy Chung, whose suggestions and insights contributed immeasurably to the development of this work. Any errors or inconsistencies are my own.
} 
The generalization that arises from this data, which I will refer to as Argument Contained Ellipsis (ACE), can be stated as in (5):

\author{
Argument Contained Ellipsis \\ Ellipsis between $\mathrm{VP}_{\alpha}$ and $\mathrm{VP}_{\beta}, \mathrm{VP}_{\beta}$ contained in an argument $\mathrm{A}_{\alpha}$ of $\mathrm{VP}_{\alpha}$, is \\ licensed only if $A_{\alpha}$ is identical to the parallel argument $A_{\beta}$ of $\mathrm{VP}_{\beta}$.
}

(5) is a descriptive statement reflecting a law of English grammar, but it is unclear why argument identity should hold in just these configurations. Ideally, this constraint should follow directly from the architecture of a theory of VP ellipsis.

The goal of this paper is to demonstrate that (5) follows from a fundamental property of anaphoric relations: dependence. A dependence relation is the relation that holds between some element (possibly silent) of linguistic structure that cannot be independently interpreted and some other entity in the discourse that provides the dependent element with a full interpretation. Specifically, I will show that the interaction of two types of dependence relations is responsible for the argument identity constraint in Argument Contained Ellipsis. The first is the relation that holds between an anaphoric element in an argument position and the linguistic element from which it receives an interpretation (its antecedent), a relation that I will refer to as referential dependence (cf. Higginbotham 1983, Haïk 1985, 1987). Intuitively, the interpretation of an anaphoric element is determined by the composition of its antecedent; hence an anaphoric element is dependent on anything contained in its antecedent. For example, in (6), the pronoun he, when interpreted as coreferential with the matrix subject, is dependent on the DP those dogs. In order to determine the value of the antecedent of the pronoun, and hence the value of the pronoun, we must know the value of those dogs.

(6) [The man who owns those dogs $]_{i}$ says he $_{i}$ takes good care of them.

The second relevant dependence relation is the one that holds between an elided verb phrase and the overt VP from which it receives its interpretation. I will refer to this relation as elliptical dependence. The status of elliptical dependence as a relation distinct from other types of anaphoric dependencies has been discussed at various points in the past (for example, in Grinder \& Postal 1971, Hankamer \& Sag 1976, and Sag \& Hankamer 1984), but it is not directly represented in recent theories of ellipsis.

Since Sag (1976) and Williams (1976), it has generally been accepted that ellipsis is licensed by an identity relation between VPs which holds at some level of semantic representation. Subsequent analyses of VP ellipsis within the Extended Standard Theory have pursued the idea that identity can be stated in terms of a syntactic level of Logical Form (LF) (e.g., May 1985, Kitagawa 1991, Fiengo and May 1993), while more purely interpretive approaches, in which the resolution of ellipsis is carried out completely in the semantic component, have followed Sag in stating identity in terms of logical expressions (e.g., Rooth 1981, Dalrymple, Shieber, \& Pereira 1991). In each of these analyses, the extent to which an elided VP can be said to be dependent on an antecedent VP is the identity relation that governs the construction of the interpretation of the elided VP (whether a syntactic or semantic representation). This relation is loose, because a common aspect of the identity requirement in these models is that it permits variation of indexical (in the syntactic accounts) or variable (in the semantic accounts) values across related VPs. Once the representation of an elided VP has been constructed, there is no fact about its structure to indicate that its interpretation is dependent on some other VP in the discourse. Elliptical dependence is not directly encoded into the representation of ellipsis.

In this paper, I will claim that the elliptical dependence relation should be explicitly represented in a theory of ellipsis interpretation, and I will demonstrate that only in such a model 
can the Argument Contained Ellipsis data be given a uniform analysis. I will propose that elliptical dependence be incorporated into a model of ellipsis by treating elliptically related VPs as identical occurrences of a single VP. That is, similarly to the approaches listed above, I will state elliptical dependence in terms of an identity relation. In contrast to the above approaches, however, I will claim that the identity relation that must hold between elliptically related VPs is strict, where strict identity forbids variation of indexical or variable value across elliptically related VPs. The reason behind this move is to incorporate into the architecture of a theory of ellipsis the following claim: ellipsis involves the reuse of linguistic material, material which is treated by other components of the grammar as identical to the original. ${ }^{1}$ This, I claim, is the nature of elliptical dependence.

I will show that it is this aspect of ellipsis that is responsible for the argument identity constraint in Argument Contained Ellipsis. Dependence relations are determined with respect to semantic representations, and are subject to certain restrictions. In particular, referentially circular constructions must be ruled out. This can be accomplished by stipulating that referential dependence relation is irreflexive: no element may be referentially dependent on itself (Higginbotham 1983, Haïk 1987, and "the i-within-i condition", Chomsky 1981). I will demonstrate that by treating elliptically related VPs as identical occurrences of the same VP, configurations in which one of two elliptically related VPs is contained in an argument of another give rise to circular constructions unless argument identity obtains. In this way, the descriptive constraint (5) will be explained in terms of a general semantic constraint that rules out structures that contain circular dependencies.

The representations of referential and elliptical dependencies, and the model of ellipsis in which these will be cast, will be stated in terms of the principles and assumptions of Government and Binding Theory (GB) (Chomsky 1981, 1986a,b). In GB, the input to the semantic component is a syntactic representation of Logical Form (LF) that is derived transformationally from $\mathrm{S}$ structure. I thus assume a syntactic model of ellipsis interpretation, in which an elided VP is fully represented at LF. The basic claims of the paper, however, regarding the nature of elliptical dependence and its interaction with other types of anaphoric dependencies, should be theory independent, and could in principle be recast in other theoretical frameworks and representations of ellipsis.

The paper is organized as follows. $\$ 2$ gives a detailed presentation of the Argument Contained Ellipsis data. $\$ 3$ sketches a model of ellipsis that provides a means of directly representing both referential and elliptical dependence; the former through the model of anaphoric relations developed in Reinhart (1983) and modified in Heim (1992), and the latter by imposing a strict identity requirement on VP ellipsis. Drawing on the work of Higginbotham (1983) and Haïk $(1985,1987)$, in §4 I discuss referential dependence, propose some modifications in line with the system of anaphoric relations adopted in $\S 3$, and state conditions on dependence. $\$ 5$ lays out my assumptions regarding the semantics of relative clauses. Finally, $\$ 6$ demonstrates that, given the machinery adopted in the previous sections, the facts of Argument Contained Ellipsis follow from the interaction of elliptical and referential dependence.

\section{Argument Contained Ellipsis}

VP ellipsis is characterized by the apparent deletion of a full VP under identity with some VP in the discourse. This phenomenon is illustrated by the examples given in (7-9), in which the missing VP is represented by the symbol [e].

(7) Fedka loved Catherine the Great but Lyosha didn't [e].

\footnotetext{
${ }^{1}$ The characterization of VP ellipsis as the "reuse of linguistic material" was suggested by Bill Ladusaw (p.c.).
} 
(8) Lena wants to move to Rome but her husband doesn't expect to be able to [e].

(9) Phyllis should have brought some artichokes, and you should have [e], too.

VP ellipsis is not limited to conjoined structures, as shown by (10-12):

(10) If John plays well, Lou does [e] too.

(11) Sterling will try the punch after Maureen has [e].

(12) Mo likes every song that Lou does [e].

The data that I will be investigating in this paper form a subset of non-conjoined elliptical structures, in which one of two elliptically related VPs is contained in an argument of the other. As noted in (5), if this relation obtains, the containing argument must be identical to the parallel argument of the contained VP. (7-12) illustrate that this identity requirement does not hold in general. The following section is devoted to demonstrating that the identity requirement is enforced in a variety of structures in which the structural configuration described in (5) does hold.

\subsubsection{Matrix Subjects}

As shown by (1) above, sentences in which an elliptical relation holds between a matrix VP and a VP embedded in a clausal complement of the head of the subject are ungrammatical. (13) and (14) further illustrate this paradigm.

(13) *Every claim that her theory is incorrect is [e].

(14) *The belief that we are mistaken is [e].

There are similar constructions in which an elliptical relation may hold between a matrix VP and a VP embedded in it's subject, however. Examples in which the subject contains a restrictive relative clause are grammatical just in case the subjects of the related VPs are identical. ${ }^{2}$ Consider the following sentences:

(15) Every man who wants to buy some salmon should [e].

(16) *Every man who wants George to buy some salmon should [e].

(17) The woman who thought she would catch a tuna did [e].

(18) *The woman who thought the man from Portland would catch a tuna did [e].

(19) A man who promised Horace to leave town did [e].

(20) *A man who persuaded Horace to leave town did [e].

In each pair, when the subject of the non-elided VP is distinct from the subject of the elided VP, the result is an ungrammatical sentence. ${ }^{3}(19)$ and (20) are a particularly interesting minimal pair. In

\footnotetext{
${ }^{2}$ For now, I will use the term "identical" informally, without giving an explicit definition, and will assume that the basic idea is fairly intuitive: in the case of referring DPs, the subjects of the related VPs should corefer; in the case of quantified DPs, the assignments of values to the subjects should be the same. An explicit statement of this idea, which will play an important role in the development of the analysis, will be developed in $\S 6$.

${ }^{3}$ The judgments of these sentences reflect the responses of a majority of informants, but it should be noted that some speakers do not find the examples in which subject identity does not hold to be robustly ungrammatical. In particular, for many people, addition of "too" or "instead" to the end of the
} 
(19), which contains the subject control verb promise, the subjects of the related VPs are identical. (20) on the other hand, contains the object control verb persuade. In this case, the subject of the embedded VP is interpreted as coreferential with the object of persuade: the DP Horace. The subjects of the related VPs are not identical, and the sentence is ungrammatical. The judgments are the same regardless of whether the elided VP is the embedded or the matrix VP.

(21) Every man who wants to [e] should buy some salmon.

(22) *Every man who wants George to [e] should buy some salmon.

(23) The man who promised Horace to [e] left town.

(24) *The man who persuaded Horace to [e] left town.

The non-elided counterparts of these sentences are grammatical, as shown by (25-27).

(25) Every man who wants George to buy some salmon should buy some salmon.

(26) The woman who thought the fisherman would catch a tuna caught a tuna.

(27) The man who persuaded Horace to clean the fish cleaned the fish.

These generalizations hold for other types of subjects as well. An elliptical relation between a matrix VP and a VP embedded in a sentential subject is impossible, as shown by (28).

(28) *That you think that their actions are surprising isn't [e].

An elliptical relation is possible, though, when the subject is a free relative clause:

(29) What you think is surprising isn't [e].

Examples like (29) are grammatical only when the free relative is interpreted as the subject of both the matrix and embedded VPs, however. (30), in which the embedded VP has a distinct subject, is ungrammatical.

(30) *What I thought would persuade Stanley to change his mind didn't [e].

In (29), the entity referred to by the free relative is the subject of both the matrix and embedded VPs. In (30), however, the free relative is interpreted as the subject of the elided matrix VP, but the subject of the embedded VP is Stanley, and the sentence is ungrammatical.

ungrammatical examples tends to make them somewhat more acceptable, although the majority of my informants still consider them unacceptable.

(i) *?Every man who wants George to buy some salmon should too.

(ii) *?The woman who thought the man from Portland would catch a tuna did instead.

There is no comparable improvement in acceptability with the addition of "too":

(iii) *A proof that God exists does too.

As has been noted frequently, VP ellipsis is extremely sensitive to contextual factors. It is possible that the improvement in acceptability of examples like (i) and (ii) results from the fact that words like "too" and "instead", in these constructions, force the hearer to assign an interpretation to what is nevertheless an ill-formed construction. See Hankamer (1978) and Higginbotham (1985) for relevant discussion of this issue. 


\subsubsection{Embedded Subjects}

The identity requirement holds of structures in which one of two elliptically related VPs is contained the subject of a clausal complement of the other, for example, in cases of Antecedent Contained Deletion (ACD) in embedded nonfinite clauses (31-32) and small clause structures (33$34)$.

(31) Mona wants the candidates that Jack does [e] to be successful.

(32) *Mona wants the rivals of the candidates that Jack does [e] to be successful.

(33) Max considers every senator Hector does [e] corrupt.

(34) *Max considers the aides of every senator Hector does [e] corrupt.

These examples are somewhat more complex that those involving identity of matrix subjects, because they involve an elliptical relation between VPs that contain embedded clauses. (31-34) do not involve identity of arguments of the elliptically related higher VPs, but rather identity of arguments of embedded VPs. The basic configuration is shown in (35), where $\mathrm{VP}_{\alpha}$ and $\mathrm{VP}_{\beta}$ are the elliptically related VPs, and XP is some clausal constituent.

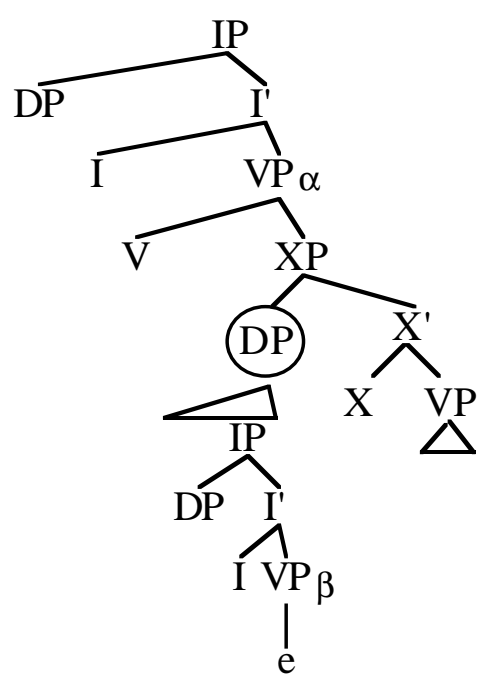

As (31-34) demonstrate, the interpretation of the circled DP must be the same in both the elided and non-elided VPs. Examples like (32) and (34), in which the embedded subject contained in the elided VP is not identical to the embedded subject contained in the matrix VP, are ungrammatical.

As in the case of matrix subjects, the non-elided counterparts of these sentences are grammatical.

(36) Mona wants the rivals of every candidate that Jack wants to be successful to be successful.

(37) Max considers the aides of every senator Hector considers corrupt corrupt.

\subsubsection{Internal Arguments}

The identity requirement holds when the elided VP is contained in an internal argument of the antecedent VP, as illustrated by the following ACD examples.

Polly visited every town Eric did [e]. 
*Polly visited every town in every country Eric did [e].

(40) Henry read each book that Fedja did [e].

(41) *Henry read each chapter of every book that Fedja did [e].

(42) Max fooled none of the senators that Hector will [e].

(43) *Max fooled none of the aides of the senators that Hector will [e].

The interpretation of (38) is given by the semantic expression $(\forall x$ :town $(x))($ Eric visited $x)($ Polly visited $\mathrm{x}$ ): for every town that Eric visited, Polly visited it, too (similarly for (40) and (42)). The ungrammatical reading of (39) is the one represented by the semantic formula $(\forall \mathrm{x}: \operatorname{country}(\mathrm{x})(\forall \mathrm{y}: \operatorname{town}(\mathrm{y}) \&$ in $(\mathrm{y}, \mathrm{x})))($ Eric visited $\mathrm{x})($ Polly visited $\mathrm{y})$ : for every country that Eric visited, Polly visited all of its towns (similarly for (41) and (43)). As noted above, in the ungrammatical examples, the elliptically related VPs have nonidentical internal arguments. ${ }^{4}$ (4446), which do not involve ellipsis, are grammatical.

(44) Sally visited every town in every country Jerome visited.

(45) Henry read each chapter of every book that Fedja read.

(46) Max fooled none of the aides of the senators that Hector will fool.

One could argue that these data are not surprising: since it is true that ellipsis is licensed by some sort of identity relation between VPs, and internal arguments are contained within the VP, internal arguments of elliptically related VPs must always be identical. Although this claim is true in general, it is not an absolute fact. An example like (47), first noted in Hirschbühler (1982), has an interpretation in which the phrase most houses in the first conjunct refers to a different set of houses from its counterpart in the interpretation of the second conjunct, thus the internal arguments of the related VPs are not identical.

A Canadian flag flew over most houses and an American one did too.

Similarly, in (48), which involves wh-movement of a direct object out of the VP, and (49), in which the related VPs are in parallel relative clauses, the internal argument of the elided VP is not identical to the internal argument of the antecedent VP.

(48) I know which senators Polly interviewed and which congressmen Eric did.

(49) I'll interview everyone Max robbed if you interview some of the people that Molly did.

Examples like these demonstrate that it is not an absolute property of VP ellipsis that internal arguments are identical, though it may be a common one. ${ }^{5}$

\footnotetext{
${ }^{4}$ I only give examples of direct objects here. As shown in the introduction, similar facts hold of indirect objects as well.

${ }^{5}$ These data are highly problematic for a theory of ellipsis that incorporates some notion of Sag's (1976) constraints on alphabetic variance. Generally speaking, VPs that contain variables bound by different VP-external operators, which is the case in (48) and (49), are not alphabetic variants. It is an interesting property of the analysis of ellipsis that will be developed here that these and similar examples are expected to be grammatical (the Hirschbühler sentence may still be problematic). I will not discuss these issues in great detail, but I will return to them briefly in $§ 7$.
} 


\subsection{The Intractability of ACE}

Clearly, the most interesting and enlightening analysis of the data presented above is one that provides a general account of the entire array of facts--that is, one that accounts uniformly for the fact that both external and internal arguments of elliptically related VPs are subject to an identity requirement when one of the related VPs is contained in the parallel argument of the other. Ideally, this constraint should follow from the structural characteristics of the sentences presented above and from the interaction of a theory of ellipsis with other components of the grammar.

The argument identity constraint in ACE structures is not generally explained within current models of ellipsis, however. Although syntactic analyses such as Kitagawa (1991) and Fiengo \& May (1993) are able to account for a subset of the data--the cases involving Antecedent Contained Deletion, in particular--these models predict that sentences such as (1) and (2b)--those in which an elliptical relation holds between a VP and a second VP embedded in the subject of the first--should be grammatical. ${ }^{6}$

The same generalization holds of Sag's (1976) deletion analysis, and current interpretive approaches such as Dalrymple et. al. (1991): these models do not predict the identity requirement in the case of subject containment. These analyses of ellipsis involve use of the lambda calculus. In Sag (1976), deletion is licensed iff the lambda expression corresponding to the meaning of a deleted VP is an alphabetic variant of the lambda expression corresponding to the meaning of some other VP in the discourse; in Dalrymple et. al. (1991), a sentence containing an elided VP is interpreted by determining the property (a lambda expression) predicated of its subject. Crucially, there is no connection (other than the predicate-argument relation) between the subject and the lambda expression, and therefore no way to place constraints on subject identity across related VPs.

There is a potential explanation for the ACE facts in the theory developed in Dalrymple et. al. (1991), however. In this theory, the interpretation of ellipsis involves two separate tasks. First, parallelism between the structurally expressed arguments of an elided VP and the arguments of an overt VP is determined; second, an equation that determines what property is predicated of the subject of an elided VP is solved. The second part of this process is crucially dependent on the first: in order to find an appropriate interpretation for a sentence containing an elide VP, it is necessary to determine which arguments in the discourse are parallel to the arguments of this VP. Building on this aspect of the theory, one could devise an explanation for the facts presented in the previous section along the following lines: it is impossible to determine parallelism between the arguments of two elliptically related VPs when the structural configuration described in (5) obtains, unless the parallel arguments were identical (cf. Kennedy 1993). The burden placed on such an analysis would be show that it was not a restatement of (5); i.e., to avoid being a descriptive generalization, not an explanation. The question asked in the introduction could be rephrased: why is argument identity required in ACE constructions in order to determine parallelism?

If the above characterization of the issues facing a parallelism-based approach is correct, however, then the analysis that I will propose in this paper can be thought of as an answer to this question as well as the one posed in the introduction. In attempting to explain the argument identity requirement in terms of the interaction of dependence relations in elliptical constructions, I take dependence, as defined in the introduction, as a fundamental, theory-independent aspect of anaphoric relations. If this claim is correct, then the results of this work should be able to be implemented in any model of ellipsis.

As noted above, two types of dependence relations play a crucial role in ACE configurations: elliptical dependence--the relation between an elided VP and the VP from which it receives its interpretation, and referential dependence--the relation between a nominal anaphoric

\footnotetext{
${ }^{6}$ For a discussion of these accounts and their predictions with respect to ACE, see Appendices A and $\mathrm{B}$.
} 
element and its antecedent. In $\S 4$, I will discuss those aspects of referential dependence that are crucially involved in Argument Contained Ellipsis. In the next section, I will propose a means of directly representing elliptical dependence in a syntactic model of VP ellipsis.

\section{Elliptical Dependence}

Since Sag (1976) and Williams (1977) it has generally been accepted that the licensing and interpretation of elliptical structures is controlled at some level of semantic representation. The theories of ellipsis articulated in Sag (1976) and Williams (1977) were conceptually very similar, differing primarily in that Sag's approach defined the conditions licensing VP deletion, whereas Williams assumed that ellipsis involved the interpretation of empty syntactic structure corresponding to a null VP by copying the semantic interpretation of an antecedent VP (cf. Wasow's (1972) Empty Structures Hypothesis). Both approaches shared the hypothesis that the conditions governing ellipsis were stated in semantic terms.

Subsequent work in GB (e.g., May 1985, Chao 1988, Kitagawa 1991, Fiengo and May 1993) has assumed that the interface between the semantic component and the syntactic component occurs at a syntactic level of Logical Form (LF), which is derived transformationally from Sstructure. These analyses claim that, given the assumption that certain well-formedness constraints apply at LF, the phenomena exhibited by elliptical structures can be shown to follow from the assumption that ellipsis involves the "reconstruction" of a null VP at LF, a process that is controlled by the syntactic form of the antecedent VP. The resulting syntactic structure is then subject to LF well-formedness constraints. Reconstruction based accounts of ellipsis differ from deletion based accounts on issues related to the organization of the grammar, but agree on the basic hypothesis that ellipsis involves syntactic representation of an elided VP at some level of structure. ${ }^{7}$ This is the position that I will adopt in this paper, though I will remain agnostic as to whether ellipsis involves reconstruction of syntactic material at LF or deletion of syntactic material at Phonological Form (PF). ${ }^{8}$

The crucial difference between previous syntactic accounts of ellipsis and the one to be proposed here is that these accounts license ellipsis through a loose identity relation between the semantic representations of related VPs. That is, although all syntactic accounts of ellipsis agree that the related VPs must have the same basic syntactic structure, loose identity accounts permit variation--within defined parameters--in the value of syntactic indices or variables across related VPs. The net result of loose identity is that the elliptical dependence relation--the connection between the elided VP and the antecedent--is not explicitly represented at LF. At the syntaxsemantics interface, the input to the interpretive component is a syntactic structure in which the surface-null VP and the VP from which it receives its interpretation are distinct. Although the related VPs have the same basic structure (up to permitted indexical variation), there is no fact about them indicative of the elliptical relation which could interact in a crucial way with some other component of the grammar. My claim is that the ACE data can be uniformly explained only if

\footnotetext{
${ }^{7}$ Arguments in favor of this hypothesis, which include the inability of ellipsis to be licensed by context (in the absence of a linguistic antecedent), parallelism requirements between the elided and antecedent VPs, and "missing antecedents" facts, are presented in detail in Hankamer \& Sag (1976). See Dalrymple (1991) for counterarguments, however.

${ }^{8}$ Let us assume that sentence can be construed as an ordered set of structural descriptions $\left\langle\mathrm{SD}_{1}\right.$, $\mathrm{SD}_{2}, \ldots, \mathrm{SD}_{\mathrm{n}}>$. Under this interpretation, a licensing condition such as the one proposed below states that a null VP is licensed in the phonological representation $\mathrm{SD}_{\mathrm{j}}$ of some sentence only if it is present in that sentence's Logical Form $\mathrm{SD}_{\mathrm{i}}$ and is identical to a VP in the logical form $\mathrm{SD}_{\mathrm{i}}{ }^{\prime}$ of some sentence already in the discourse. In principle, the necessary relation could obtain either through deletion of syntactic material at PF or through reconstruction at LF; to maintain generality, I will not attempt to choose one view over the other in this paper.
} 
there is an explicit representation of elliptical dependence at LF, allowing it to interact with other components of the grammar; specifically, with other dependence relations. In the following section, I will propose a way of incorporating elliptical dependence into a syntactic model of ellipsis.

\subsection{Strict Identity in VP Ellipsis}

In $\S 1$, I claimed that ellipsis involves the reuse of linguistic material, and that the elliptical dependence relation reflects this "recycling". I propose that this relation be incorporated into a syntactic theory of ellipsis by imposing the following licensing condition on ellipsis, where strict identity means that indexical values are constant across elliptically related VPs: ${ }^{9}$

(50) A null VP $\alpha$ is licensed at PF only if there is some VP $\beta$ in the discourse such that $\alpha$ 's LF is identical to $\beta$ 's LF.

Consider the implications of this statement, which I will refer to as the strict identity hypothesis. The claim that elliptically related VPs are strictly identical entails that all syntactic indices that appear in the LF representation of an antecedent VP must be reproduced exactly in the LF representation of an elided VP. Given these assumptions, it follows that a sentence like (51) has the LF shown in (52). ${ }^{10}$

(51) Leo drives a Dart and Felix does too.

(52) Leo ${ }_{1}\left[\mathrm{vP} \mathrm{t}_{1}\right.$ drives a Dart] and Felix ${ }_{1}$ does [vP $\mathrm{t}_{1}$ drive a Dart] too

A structure like (52) would not arise in loose identity models of ellipsis for two reasons. First, in e.g. Kitagawa (1991) and Fiengo \& May (1993), the VP internal subject trace is just the type of anaphoric element whose indexical value may vary across elliptically related VPs. Second, in these models, coindexation entails coreference, so a structure like (52) could only have an interpretation in which the names Leo and Felix refer to the same individual. The more normal reading, in which the names refer to distinct individuals, would be unavailable.

(52) is a specific example of a general result of the proposed model of ellipsis: in order to satisfy the LF requirement that the trace in the specifier of an elided VP must have a coindexed antecedent, the subjects of elliptically related VPs must bear identical syntactic indices. A Logical Form in which this relation does not obtain, such as (53), would be ruled out by independent principles of the grammar (cf. fn. 10).

$$
\text { Leo }_{1}\left[\mathrm { vP } \mathrm { t } _ { 1 } \text { drives a Dart] and Felix } { } _ { 2 } \text { does } \left[\mathrm{vP}_{\mathrm{t}} \mathrm{t}_{1}\right.\right. \text { drive a Dart] too }
$$

\footnotetext{
${ }^{9}(50)$ is very similar to Sag's (1976) statement of VP Deletion; the main difference between this approach and Sag's is that (50) requires strict identity, whereas Sag required alphabetic variance of lambda expressions, a form of what I have referred to as loose identity.

${ }^{10}$ I assume the VP internal subject hypothesis (Kitagawa 1986, Koopman \& Sportiche 1988, Kuroda 1988), and in particular, the VP structure defended in Huang (1993), in which the subject originates in SpecVP and moves to SpecIP to receive Nominative Case. Moreover, I assume SpecVP to be the position to which $\mathrm{V}^{0}$ assigns its external theta role. Any model of ellipsis that utilizes a syntactic, identity-based theory of VP reconstruction must account for the fact that all VP internal traces must have antecedents (a requirement that can be formalized in a number of ways, e.g., the Empty Category Principle, the definition of chain). In particular, the well-formedness conditions imposed on the subject trace in the specifier of the antecedent VP must be met by the parallel trace in the specifier of the LF representation of the elided VP.
} 
Clearly, if the strict identity model is to be accepted, a distinction must be made between coindexation, coreference, and the type of relation that holds between traces and their antecedents, in order to ensure that structures such as (52) are both well-formed with respect to syntactic constraints and interpretable in a normal way.

Precisely this distinction is made in the model of anaphoric relations developed in Reinhart (1983). A fundamental claim of this theory is that coindexation does not entail coreference, nor does non-coindexation entail non-coreference. Indices--numerical subscripts on DPs--represent only syntactic binding relations. The central claim of Reinhart's approach to anaphora is that bound variable anaphora and coreference are fundamentally different: whereas bound variable anaphora is dependent on structural relations between constituents and should be represented in the syntax, coreference is essentially a discourse-dependent relation that is controlled by pragmatic factors. In this framework, the role played by syntactic indices in the determination of reference is substantially different from that in other models of anaphoric relations GB, such as Fiengo \& May (1993), in which coindexation entails coreference. For Reinhart, indices represent only binding relations; they do not indicate coreference.

In this system, then, there is no requirement that the like-indexed subjects of the elliptically related VPs in (52) (repeated below) corefer.

(52) Leo 1 [vp $\mathrm{t}_{1}$ drives a Dart] and Felix ${ }_{1}$ does $\left[\mathrm{vP}^{\mathrm{t}} \mathrm{t}_{1}\right.$ drive a Dart] too

(52) is a perfectly well-formed LF. The indices on the subjects of the elliptically related VPs indicate which elements within their respective c-command domains are to be interpreted as variables bound by them. That is, the coindexed subjects bind all and only like-indexed anaphoric elements within their respective scopes; the interpretation of these anaphoric elements is governed solely by their binders, not by the numerical values of syntactic indices (Reinhart 1983, Heim 1992, Grodzinsky \& Reinhart 1993). This fact can be clearly demonstrated if the semantic translation of (52) is represented using lambda notation, substituting the same letter, "x", for the numerical index "1", as in (54):

\section{(54) Leo $\lambda x(x$ drives a Dart $)$ and Felix $\lambda x(x$ drives a Dart $)$}

In (54), the interpretation of the variable bound by the lambda operator in each conjunct is governed solely by the scope of the lambda operator that binds it, not by the alphabetic value of the variable itself.

Although Reinhart's system provides a framework of anaphoric relations in which the strict identity model of ellipsis can be implemented, it does not provide a formal means of indicating coreference. As noted above, coreference in Reinhart's model is not encoded in the formal representation of a sentence, rather it is pragmatically determined. There is no way to state grammatical constraints on coreference relations in terms of syntactic indexing configurations, because these types of indexing configurations do not exist. Only binding relations are indicated by syntactic indexing. In $\$ 4$, we will see that both coreference and bound variable anaphora are involved in referential dependence relations, and that constraints on possible referential dependence relations can be clearly stated in terms of constraints on indexing configurations at Logical Form. As I intend to show that the Argument Controlled Ellipsis facts follow from the interaction of elliptical dependence and referential dependence, it would be to my advantage to employ a system of anaphoric relations that formally encodes both binding and coreference. Heim (1992) develops a revision of Reinhart (1983) which does exactly this. Heim's system captures the formal distinction between bound variable anaphora and coreference, but also allows coreference relations to be represented in the syntax. In order to ensure maximal clarity of the presentation of the analysis in $\S 6$, I will adopt Heim's revision of Reinhart in this paper. In the following section, I will give a 
brief introduction to Heim (1992), then show how the Logical Form of a sentence involving VP ellipsis is constructed within the more articulated theory of indexing proposed by Heim.

\subsection{Indices, Binding, and Coreference}

Heim (1992) makes the distinction between bound variable anaphora and coreference formally precise by encoding both types of relations into the structural representation of sentences via syntactic indexing configurations. This task is accomplished by allowing DPs to receive two indices, "an inner index that encodes what they are bound by, and an additional index that encodes what they in turn bind.... The inner and outer index need not be the same" (Heim 1992:37). (As Heim notes, such a system has a precedent in the linking framework of Higginbotham 1983, a connection that I will return to below.) Indexing relations are defined as in (55) and (56), where linking is the relation relevant to bound variable anaphora, and colinking is the relation relevant to coreference. For reasons that will become clear in $\$ 4.1$, having to do with issues of referential dependence and referential circularity, I have modified Heim's definitions in order to explicitly state that these relations are irreflexive. ${ }^{11}$

(55) Def $\alpha$ is linked to $\beta$ iff $\beta$ 's outer index equals $\alpha$ 's inner index and $\alpha \neq \beta$.

(56) Def $\alpha$ and $\beta$ are colinked iff $\alpha$ 's inner index equals $\beta$ 's inner index and $\alpha \neq \beta$.

As noted by Bill Ladusaw (personal communication), the effect of this indexing system is to represent the distinction between a variable and the name of a variable in the syntactic structure. Intuitively, a "variable" is an anaphoric element whose interpretation is set by some outside operator or function, while a "name of a variable" is a diacritic on an operator that indicates which variables within a certain domain (e.g., the operator's scope) that operator may bind. This distinction is illustrated by the predicate logic formula in (57).

$$
\forall \mathrm{x}[\mathrm{P}(\mathrm{x}) \& \mathrm{Q}(\mathrm{x})]
$$

In (57), there are two occurrences of the variable $x$ : as arguments of the predicates $\mathrm{P}$ and $\mathrm{Q}$. The occurrence of $x$ to the immediate right of the universal quantifier is not a variable, but rather a diacritic indicating which variables may be bound by the quantifier. Heim's indexing system directly parallels this example. Inner indices correspond to variables in the sense described above, and outer indices are diacritics indicating the "binding potential" of a particular DP.

Crucially, outer indices do not play a role in determining the referential value of the DP to which they are associated, a fact which will be important to the implementation of the strict identity hypothesis in $\S 3.3$. The function of an outer index is to indicate that a particular DP has operator status and to indicate which indices within that DP's scope are to be interpreted as variables bound by it. Outer-indexed DPs in Heim's system are precisely those DPs in Reinhart (1983) that are antecedents in cases of bound variable anaphora; that is, those DPs that c-command a coindexed pronoun. In Reinhart (1983), both the variable status of a pronoun and the operator status of a DP are represented by the same type of indexing notation. The effect of Heim's notation is to distinguish between indices that correspond to variables (inner indices), and indices that indicate the operator status of a DP (outer indices).

${ }^{11}$ There are three logically possible indexing configurations: inner only, outer only, inner and outer. I will represent these configurations in the following way: inner only $=\mathrm{X}_{i}$, outer only $=\mathrm{X}_{: i}$, inner/outer $=X_{i: j}$. 
With this understanding of the formal notation, we can make the following claims about the interpretation of linking and colinking configurations. 12

A node $\alpha$ is bound by a node $\beta$ iff $\alpha$ is linked to $\beta$ and $\beta$ c-commands $\alpha$. Translation Definition Inner indices are translated as variables at LF.

These relations are illustrated by the Logical Form in (60).

$$
\text { Leo }_{2: 1}\left[\mathrm{vP} \mathrm{t}_{1} \text { likes his }_{2}\right. \text { Dart] }
$$

In (60), the VP internal trace is linked to and c-commanded by the subject Leo, and so is bound by this DP. The pronoun his is not linked to the subject, but these DPs are colinked. In Heim's system, colinking--identity of inner indices--is the indexing relation relevant to coreference. Given the translation definition (59), it follows that an occurrence of an inner index that is not ccommanded and linked to some outer index must be a free variable. For an LF to be interpretable, all free variables must receive referents. This task is accomplished by a rule of reference assignment which requires all occurrences of a particular free variable to denote the same individual (cf. Heim 1992:53). According to this rule, the LF in (60) is interpretable just in case the colinked DPs Leo and his corefer.

A detailed explication of the colinking/linking system is beyond the scope of this paper; I refer the interested reader to Heim (1992) for a discussion of how incorporating these relations into the syntax allows her to account for a range of traditionally problematic data (e.g., apparent Condition B violations). It is important to note, however, that this system departs from the concept of syntactic indexing assumed in Reinhart (1983). In particular, it departs from Reinhart's claim that coreference is not formally represented in the syntax by syntactic indexing. Heim's rule for interpreting colinked structures means that coindexation entails coreference: every occurrence of a particular free inner index must be associated with the same referent. Although there is nothing to prevent different inner indices from being associated with the same referent (as in Reinhart 1983), it cannot be the case that the same free index is associated with different referents.

To summarize, all inner indices correspond to free variables. Outer indices are diacritics that indicate which indices within a particular DP's c-command domain are to be construed as bound by that DP. This system provides a formal means of representing both binding (via linking), and coreference (via colinking). This more complex formal apparatus permits us to maintain the conceptual division between coreference and binding at the heart of Reinhart (1983), but, at the same time, allows us to impose specific grammatical constraints on each relation. This fact will be of crucial importance in the discussion of referential dependence in $\S 4$. But before we address those issues, I will discuss the representation of ellipsis under the strict identity hypothesis, given the assumptions made in this section.

\footnotetext{
12 Two things should be said about (58) and (59). First, by assuming a general constraint against vacuous quantification, (58) can be understood as including an implicit minimality requirement. That is, in configurations of the form shown in (i), where $i_{1}$ and $i_{2}$ are like-valued inner indices and $i_{2}$ is ccommanded by both $\mathrm{X}_{: i}$ and $\mathrm{Y}_{: i}, i_{2}$ is bound by $\mathrm{Y}_{: i}$, even though (58) does not literally prohibit $i_{2}$ from being bound by $\mathrm{X}_{: i}$.

(i) $\quad\left[\mathrm{X}_{: i} \ldots i_{1} \ldots \mathrm{Y}_{: i} \ldots i_{2} \ldots\right]$

Second, (59) is a modified version of Heim's original translation definition. Heim states that only definite DPs, pronouns, and traces bear inner indices. For the purposes of this paper, I will assume that indefinites may also bear inner indices, which, when bound by existential closure, introduce a discourse referent (cf. Heim 1982, Diesing 1992, and fn. 17 below). Indices on definites and indefinites are subject to the Novelty-Familiarity Condition, however (Heim 1982).
} 


\subsection{The Representation of Ellipsis}

As stated in (50), I claim that an elided VP is licensed in the phonological representation only if its Logical Form is identical to the Logical Form of some VP in the discourse. ${ }^{13}$ Following Heim (1992), I will assume that LFs are related to surface structures by an optional operation of quantifier raising $(\mathrm{QR})$, which takes any DP that bears an outer index and adjoins it to a dominating clausal node, leaving behind a trace which bears an inner index of the same value as the outer index of the moved DP. ${ }^{14}$ For the purposes of this paper, we may assume that QR does not apply unless it has to (e.g., in Antecedent Contained Deletion structures; see May 1985 and $\$ 6$ below).

In particular, I will assume that a DP in SpecIP that bears an outer index is interpreted as an operator, and the trace in SpecVP as the variable that it binds (cf. Diesing 1992). As noted in \$3.1, given the claim that the LF of an elided VP is identical to the LF of its antecedent, this requirement would seem to force coindexation of the subjects of elliptically related VPs. The situation is somewhat different now, given the assumptions regarding the representation of anaphoric relations that were made in the last section. In Heim's system, the Logical Form of a sentence like (51), repeated below, is $(61) .15$

${ }^{13}$ Due to space considerations, I will not give a detailed presentation of how various ambiguities associated with elliptical structures are to be accounted for within a strict identity model (i.e., strict and sloppy interpretations of pronouns); see Appendix $\mathrm{C}$ for a brief survey of such constructions. The analysis is essentially the same as that of Reinhart (1983), in which strict readings are correlated with interpretations of VP internal pronouns in which they are coreferential with a subject, and sloppy readings with bound variable interpretations of VP internal pronouns. The analysis proposed here will inherit any problems with such an analysis of strict/sloppy ambiguity (see Wescoat 1989 for some particularly problematic examples), but this is not an issue that I will address. The goal of this paper is not to promote a particular theory of ellipsis, but to propose that elliptical dependence be explicitly represented within a theory of ellipsis.

${ }^{14}$ According to Heim, the sister constituent of the moved DP is then prefixed with a lambda operator which bears the outer index of the moved DP. I will assume here that prefixation of the lambda operator to the sister of the moved DP is part of the process of mapping the syntactic representation of an utterance's Logical Form onto its semantic interpretation. The lambda operator is a logical entity, and presumably not overtly represented in the syntax. The scope of the lambda operator in the semantic interpretation of a given LF is predictable: it is the c-command domain of the prefixed DP.

${ }^{15}$ (61) is not the complete LF of (51). In particular, I have not shown the indices on the indefinite DPs. The full representation of the LF of (51) is given in (i).

(i) $\mathrm{Leo}_{2: 1}\left[\mathrm{VP}^{\mathrm{t}} \mathrm{d}_{1}\right.$ drives a Dart 4 and Felix 3:1 does [VP $\mathrm{t}_{1}$ drive a Dart 4 ] too

(i) reflects the fact that the strict identity hypothesis as stated in (50) requires the indefinites to bear the same inner indices. The reader may now justifiably point out that according to the claim in $\$ 3.2$ that all free inner indices are assigned the same referent, (i) should mean that Leo and Felix drive the same car. Although this is a possible interpretation of (i), there is also an interpretation in which Leo and Felix drive different cars. On the surface, it appears that the proposed analysis does not allow this reading.

There is a way out of this problem, however. The reading in which Leo and Felix drive different cars results when each occurrence of the indefinite DP a Dart in the two VPs introduces a discourse referent. In a theory of the syntax-semantics interface such as Heim (1982) or Diesing (1992), indefinites that introduce discourse referents are bound by existential closure. We can therefore say that on the relevant reading of (i), the inner indices of the two indefinites are not free, but bound by existential closure. Since these indices are not free, they do not corefer. 
Leo drives a Dart and Felix does too.

Leo $_{2: 1}\left[\mathrm{VP} \mathrm{t}_{1}\right.$ drives a Dart $]$ and Felix $3: 1$ does $\left[\mathrm{VP} \mathrm{t}_{1}\right.$ drive a Dart $]$ too

(50) states that the LF of an elided VP must be identical to the LF of some VP in the discourse. This condition is true of (61). ${ }^{16}$ Moreover, this LF is well-formed with respect to operator-variable relations: in both conjuncts, the subjects bind a trace in SpecVP. This binding relation in the second conjunct holds because the subject of the elided VP bears the same outer index as the subject of the antecedent VP. (61) should be contrasted with (62), in which the subjects of the elliptically related VPs do not bear the same outer index.

(62) $\quad$ Misha $_{2: 1}\left[v t_{1}\right.$ drives a Dart] and $\operatorname{Ivan}_{3: 4}\left[v_{P} t_{1}\right.$ drive a Dart] too

Although an elliptical relation is licensed in a structure like (62) because the VPs are strictly identical, (62) is not a well-formed LF. Assuming a general condition on LFs that subjects must bind a variable in SpecVP (see fn. 10; cf. also Full Interpretation, Chomsky 1986b), (62) is illformed because the subject of the second conjunct does not bind the trace in SpecVP.

These examples illustrate a specific result of the proposed analysis of ellipsis: the subjects of elliptically related VPs must bear the same outer indices; elliptical structures in which this indexing does not hold are uninterpretable. Such indexing configurations are perfectly compatible with Heim's system, the crucial point being that identity of outer indices does not indicate an anaphoric relation between two DPs. It is this particular aspect of Heim (1992) that allows us to maintain the claim that ellipsis involves strict identity of related VPs, as stated in (50).

The most important difference between a model of VP ellipsis based on strict identity and one based on loose identity is that in the former, elliptical dependence is explicitly represented at LF by the identity of indexical values across related VPs. This identity should be understood as a formal means of representing the proposal that the Logical Form of an elided VP is treated by the grammar as identical to the LF of the VP from which it receives its interpretation; that is, that elliptical dependence represents the reuse of linguistic material. In loose identity model, in which elliptical dependence is not directly represented, the representations of elliptically related VPs are distinct.

In most cases of ellipsis, the hypothesis that an elided VP is treated as identical to its antecedent does not have unexpected results, given a model of anaphoric relations such as Heim (1992) (or Reinhart 1983). Because two distinct DPs may bind like-valued variables (by virtue of bearing identical outer indices)--modulo configurational restrictions such as c-command--the strict identity analysis is indistinguishable from an analysis in which the indexical values of anaphoric elements within the VPs are allowed to vary. There is a set of configurations in which the strict identity and loose identity models make different predictions, however. These are configurations in which one of two elliptically related VPs is contained in an argument of the other; that is, Argument Contained Ellipsis configurations. The two models diverge in their predictions as the result of the interaction between elliptical dependence, as formalized in this section, and referential dependence, which I defined in the introduction as the relation between an anaphoric element in an argument position and its antecedent. In the following section I will present a theory of referential dependence based on the work of Higginbotham (1983) and Haï (1987), relativized to the system of anaphoric relations adopted here. In $\$ 6$ we will see that the interaction of referential and elliptical dependence accounts for the Argument Contained Ellipsis facts.

${ }^{16}$ A structure such as (i) would not be a possible LF of (51), because the VPs differ in indexical value of the traces in SpecVP.

(i) Leo $_{2: 1}\left[\mathrm{VP}^{\mathrm{t}_{1}}\right.$ drives a Dart $]$ and Felix $3: 4$ does $\left[\mathrm{VP}_{4} \mathrm{t}_{4}\right.$ drive a Dart $]$ too 


\section{Referential Dependence}

\subsection{Coreference and Binding}

As noted by Heim, her double indexing system bears a strong resemblance to the linking framework of Higginbotham (1983), a central principle of which is referential dependence. Stated roughly, an anaphoric element is dependent on its antecedent and anything contained in its antecedent. The exact formulation is given in (63) (Higginbotham 1983:404), where $\alpha$ is an antecedent of $\beta$ if $\beta$ is linked to $\alpha$ or, for some $\gamma, \beta$ is linked to $\gamma$ and $\alpha$ is an antecedent of $\gamma$.

(63) $\alpha$ is dependent on $\beta$ if:

(i) $\quad \beta$ is contained in an antecedent of $\alpha$ or

(ii) for some $\gamma, \alpha$ is dependent on $\gamma$, and $\gamma$ is dependent on $\alpha$.

This relation is irreflexive: an element must never be construed as dependent on itself. Higginbotham formalizes this condition as the following constraint on LF representations:

(64) *dependent $(\alpha, \alpha)$

This condition is designed to prohibit referential circularity: it should not be the case that some element's interpretation must be known in order to determine its interpretation. (65) is a typical example of referential circularity. ${ }^{17}$

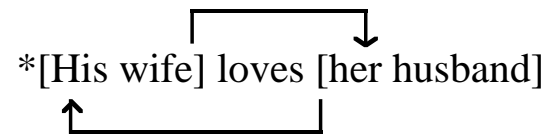

By (63), the pronoun his is dependent on the pronoun her, which is contained in the antecedent of his. The pronoun her is dependent on his, which is contained in the antecedent of her. By transitivity each pronoun is dependent on itself, in violation of (64). In addition, (64) rules out socalled $i$-within- $i$ violations (Chomsky 1981), which are exemplified by (66).

\section{*[Every picture of its frame] was taken on Wednesday.}

The antecedent of the pronoun its is the DP containing it, therefore this pronoun is dependent on itself, in violation of (64).

In Higginbotham's system, in which anaphoric relations are uniformly represented via linking, (64) is a satisfactory definition of dependence. In a system such as Reinhart/Heim's that assumes two types of anaphoric relations--bound variable anaphora and coreference--giving a specific statement of dependence becomes somewhat more complex. Some constraint against referential circularity is required in order to rule out sentences like (65) and (66); the question is, how should this relation be formulated?

Let us begin with the assumption that Higginbotham's (63) can be directly applied to Heim's system without modification. Recall that $\alpha$ is linked to $\beta$ if $\alpha$ 's inner index equals $\beta$ 's outer index. With antecedence defined as in Higginbotham (1983), the LF of (66) will be ruled

\footnotetext{
${ }^{17}$ In Higginbotham's system, anaphoric relations are represented with headed arrows. These arrows directly parallel Heim's inner and outer indices: the tail of an arrow corresponds to an inner index; the head of an arrow corresponds to an outer index.
} 
uninterpretable in Heim's system for the same reason that it is uninterpretable in Higginbotham's system: the pronoun is both linked to and contained in its antecedent. Consider (67):

(67) [every picture of its 2 frame]:2 was taken on Wednesday.

What about (65)? In Heim's system, a sentence like (65) may have an LF as in (68), in which no DP bears an outer index (these are optional).

(68) $\quad\left[\right.$ his $_{1}$ wife $_{3}$ loves $\left[\text { her }_{3} \text { husband }\right]_{1}$

His wife and her are colinked, therefore coreferential; her husband and his are colinked, therefore coreferential. Because dependency is defined in terms of antecedence, and antecedence is defined in terms of linking, according to (63), the pronouns are dependent neither on these DPs nor on anything contained within them, and (68) should not involve a violation of (64). This is clearly an undesirable result, as the intuition is very strong that (68) is uninterpretable because it involves referential circularity. How should this intuition be formally stated in a system that assumes both linking and colinking?

Clearly, my initial assumption that Higginbotham's linking directly parallels Heim's notion of linking must be modified. This is understandable since Higginbotham's linking represents both binding and coreference, whereas Heim's linking represents only binding. The fact that Heim's system represents anaphoric relations in two ways--via linking and via colinking--suggests a solution to the question raised above: if there are two types of anaphoric relations, then it follows that there are two types of "referential" dependence. ${ }^{18}$ In the following paragraphs I will present a reformulation of dependence in terms of Heim (1992).

I propose that a system of anaphoric relations that assumes both binding and coreference must in turn assume two types of dependence. The first, which I will call $r$-dependence, arises in colinked structures, i.e., in cases of discourse-controlled coreference. R-dependence is defined in $(69)^{19}$ :

\section{r-dependence}

$\alpha$ ( $\alpha$ has no descriptive content) is r-dependent on $\beta$ if:

(i) for some $\gamma, \alpha$ and $\gamma$ are colinked and $\beta$ is contained in $\gamma$, or

(ii) $\alpha$ is r-dependent on $\gamma$ and $\gamma$ is r-dependent on $\beta$.

The second type of dependence arises in binding relations. Intuitively, this type of dependence is a relation between a bound variable and the restriction of its binder: if the domain over which the variable may range is determined by the restriction, then the possible value of the variable is dependent on (the elements within) the restriction. I will call this type of dependence $b$ dependence, and define it as in (70):

\section{$b$-dependence}

$\alpha$ ( $\alpha$ has no descriptive content) is b-dependent on $\beta$ if $\beta$ is contained in the binder of $\alpha$.

18 Alternatively, we could define two types of antecedents: one with respect to linking (coreference) configurations; one with respect to binding configurations, and retain Higginbotham's definition of dependence. The end result would be the same, but would not reflect as clearly as refining the notion of dependence does the fact that we are really making a distinction between binding and coreference.

${ }^{19}$ Following Higginbotham, I assume the "contained in" relation to be reflexive. 
The crucial question is whether both of these relations, like Higginbotham's statement of dependence, are irreflexive. We can answer this question by reexamining the two examples of dependence violations given above. First consider (68), which was not ruled out by (64).

(68) $\quad\left[\right.$ his ${ }_{1}$ wife $_{3}$ loves $\left[\text { her }{ }_{3} \text { husband }\right]_{1}$

In (68), the pronoun his is colinked with the DP her husband. According to (69i), his is dependent on both this DP and the pronoun her, which is contained in the DP. Similarly, her, which is colinked with his wife, is dependent on his. By (69ii), each pronoun is dependent on itself. The fact that (68) is ungrammatical indicates that $r$-dependence is irreflexive. contains it.

We may now turn to linking structures. In (67) the pronoun is linked to the DP that

(67) [every picture of its $_{2}$ frame $_{: 2}$ was taken on Wednesday]

According to (70), because the pronoun its is contained in its binder, it is b-dependent on itself. In general terms, the pronoun is part of the restriction of its binder, which, as discussed above, is a configuration that must be ruled out. (67) and other i-within-i violations are straightforwardly explained if $b$-dependence, like $r$-dependence, is irreflexive. These conclusions allow us to reformulate Higginbotham's (64) into the following two constraints, which rule out circular structures at LF:

$$
\begin{aligned}
& { }^{*} \text {-dependent }(\alpha, \alpha) \\
& { }^{*} \text { b-dependent }(\alpha, \alpha)
\end{aligned}
$$

(71) and (72) can be implemented in the grammar as constraints on syntactic indexing configurations. These constraints reject Logical Forms that contain indexing configurations that correspond to reflexive dependence relations, with respect to the definitions stated in (69) and (70).

\subsection{Dependence Relations Within DP}

An immediate worry, pointed out in Haïk (1987), is that (72) is too strong a constraint. Crucially, it must not rule out structures such as (73), which are discussed in Haïk (1987), or cases of ordinary relative clauses, such as (74).

(73) [Every man near his ${ }_{2}$ Dart $]_{2}$ looks happy.

(74) [Every man who cleans his ${ }_{2}$ Dart $_{: 2}$ is happy.

(73) and (74) should be contrasted with the ungrammatical structures (75) and (76), both of which involve b-dependence violations. ${ }^{20}$

(75) $*$ [every picture of its 2 frame $]_{2}$ was taken on Wednesday.

(76) $*$ [every proof that it ${ }_{2}$ is correct $]_{2}$ is invalid.

20 (77) incurs a dependency violation for the same reason as (76): the pronoun it is bound by a containing DP. 
Haik (1987) observes that the difference in grammaticality between (73) and (74) on the one hand and (75) and (76) on the other follows directly from structural differences between the grammatical and ungrammatical examples. Let us assume the structure of DP to be as in (78):21

(78)

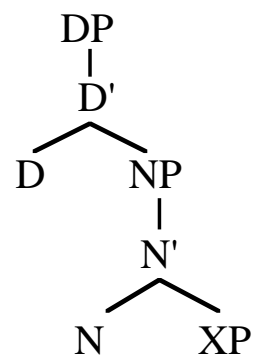

(76) and (77) contain selected complements of N--a PP in (76) and a CP in (77)--which occupy the structural position of XP in (78). In either case, regardless of whether NP or DP is the binder of the pronoun in the selected complement, the pronoun is contained in its binder and therefore $\mathrm{b}$ dependent on itself, in violation of (72). In (73) and (74), however, the phrase containing the bound pronoun is an unselected modifier. Assuming that modifiers are adjoined to the phrases they modify, they do not occur in the position of XP in (78). The adjunction prohibition (Chomsky 1986a) prohibits adjunction to an argument, therefore the PP in (73) and the CP in (74) must be adjoined to the NP complement of $\mathrm{D}$, which is the complement of a functional head and not an argument. That is, the PP and CP occupy the position represented by YP in the DP structure (79).

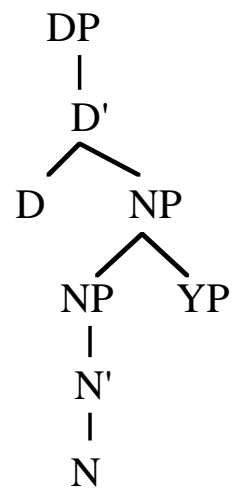

In (79), YP is contained in DP, so if DP binds an element within YP, the structure is ruled out by (72). YP is not, however, contained in the lower NP. If this NP is the binder of an element within the adjoined YP, (79) does not incur a violation of (72): the bound element is not contained in its binder, so it is not dependent on itself. ${ }^{22}$ To illustrate, the structure of the subject in (73) is given in (80):

${ }^{21}$ By allowing outer indices appear on every maximal projection in the extended projection of $\mathrm{N}$, i.e., on both DP and NP (cf. Grimshaw 1991), we can assume that both DP and NP are potential binders, modulo the c-command constraint on binding.

${ }^{22}$ Note that a structure in which the higher NP is the binder of an element inside YP is ruled out for the same reason that DP cannot be a binder--the bound element in YP would be b-dependent on itself. 


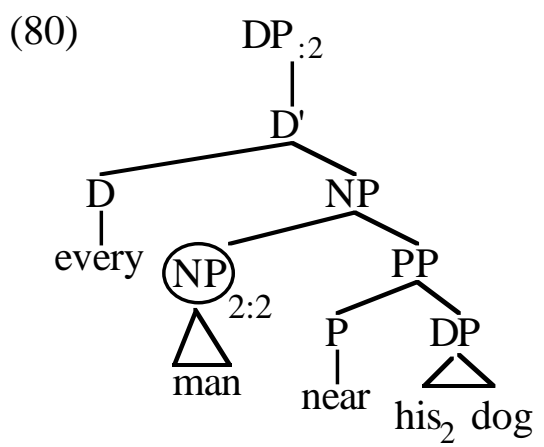

The pronoun his is bound by the circled NP--not by DP, so this structure does not violate (72).

The analysis of relative clauses is similar to that of PP modifiers, but some details must be discussed, as an account of binding relations within relative clauses will be of crucial importance to the analysis of the Argument Contained Ellipsis structures. The (abbreviated) structure of the subject relative clause in (74) is shown in (81):

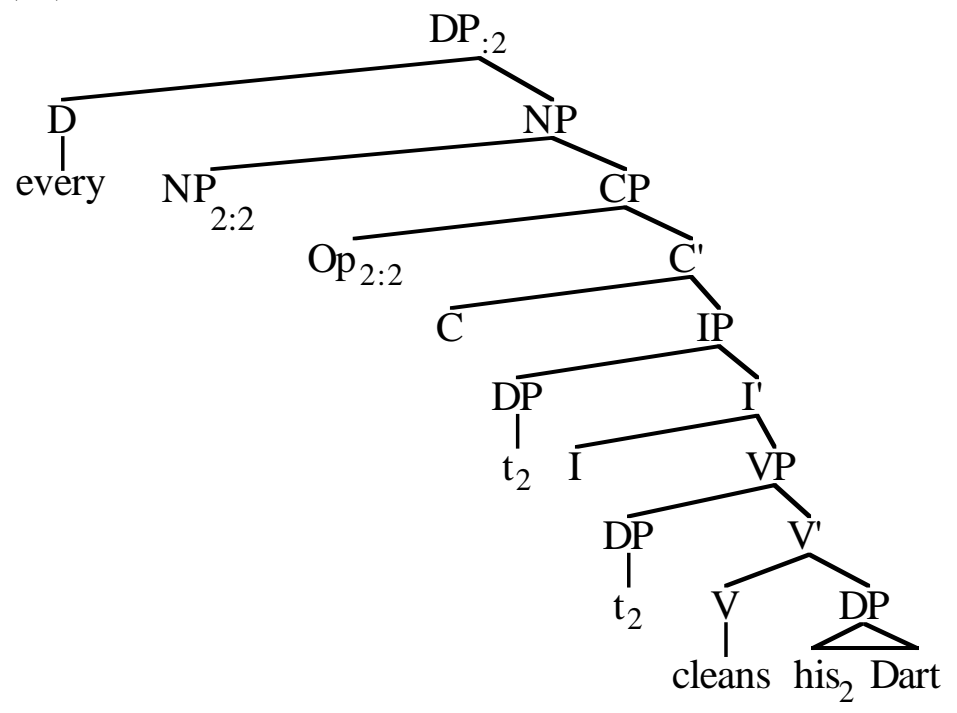

In (81), the relative operator bears an inner and outer index. This structure formally represents the binding relation that, according to Safir (1986), holds between the head of the relative clause and the relative operator. The outer index binds the traces within the $\mathrm{CP}$; the inner index is linked to the head of the relative clause, which c-commands the relative operator and therefore binds it, according to the assumptions made above. (81) does not violate (72): nothing in the relative clause is bound by an element that contains it, therefore nothing in the relative clause is b-dependent on itself.

The analysis of b-dependence presented here and its relation to i-within-i condition violations builds on that of Haïk (1987), who notices that referential circularity is blocked if an element which has the same interpretation as the XP that contains it is bound within that XP. Haïk formalizes this observation as the following principle (Haïk 1987:506): 


\author{
Principle of Referential Dependency \\ In $\left[\mathrm{XPi}_{\mathrm{i}} \ldots \mathrm{x}_{\mathrm{j}} \ldots\right]$, if $\mathrm{x}_{\mathrm{j}}$ referentially depends upon some antecedent $\mathrm{XP}_{\mathrm{j}}$, then \\ $\mathrm{XP}_{\mathrm{i}}$ is annotated as referentially dependent upon the antecedent, unless $\mathrm{x}_{\mathrm{j}}$ \\ is bound inside $\mathrm{XP}_{\mathrm{i}}$.
}

Haïk assumes a constraint on circularity identical to Higginbotham's *dependent $(x, x)(64)$; the combination of (64) and (82) rules out the ungrammatical structures discussed above, but allows the grammatical (73) and (74). The crucial difference between the analysis presented here and Haïk's analysis is that, as noted in Haïk (1985), (82) is a descriptive constraint--in a system that assumes a single referential dependence relation (as in Higginbotham 1983 and Haïk 1987), it does not follow that binding within XP should save structures like (73) and (74) from ill-formedness with respect to (64). In the current analysis, which distinguishes between dependence in binding relations and dependence in coreference relations, the well-formedness of e.g. (73) and (74) is expected. Because the anaphoric elements in these structures are bound, the relevant dependence relation is b-dependence. As illustrated by the structural representations (80) and (81), the pronouns are not contained within their binders, so are not b-dependent on themselves. The same cannot be said of (75) and (76), in which, as shown in (78), the pronouns are contained within their binders. The contrast between (73) and (74) on the one hand and (75) and (76) on the other is straightforwardly explained.

To summarize, this section claimed that within the system of anaphoric relations developed in Heim (1992), the general notion of "referential" dependence should be stated both in terms of coreference (colinking), and in terms of binding (linking). These two types of dependence were defined as $r$-dependence and $b$-dependence, respectively. By assuming that these relations are irreflexive, and irreflexivity is enforced in the form of constraints on indexing configurations at LF, we were able to account for the uninterpretability of a variety of circular constructions. In addition, it was shown that the grammaticality of a set of structures that are superficially similar to reflexive b-dependence structures, which were previously accounted for by e.g. Haïk 1987 with a descriptive constraint, follows directly from structural characteristics and the definition of b-dependence.

\title{
5 Formal Variables
}

A generalization common to the grammatical examples of Argument Contained Ellipsis presented in $\S 2.1$ (the ones in which argument identity obtains) is that the VP contained within the argument of the other VP is contained in a relative clause. Before moving to an analysis of ACE, I will first lay out some assumptions regarding the syntax and semantics of relative clauses. The structure given in (81), repeated below, can be used to illustrate the discussion. 


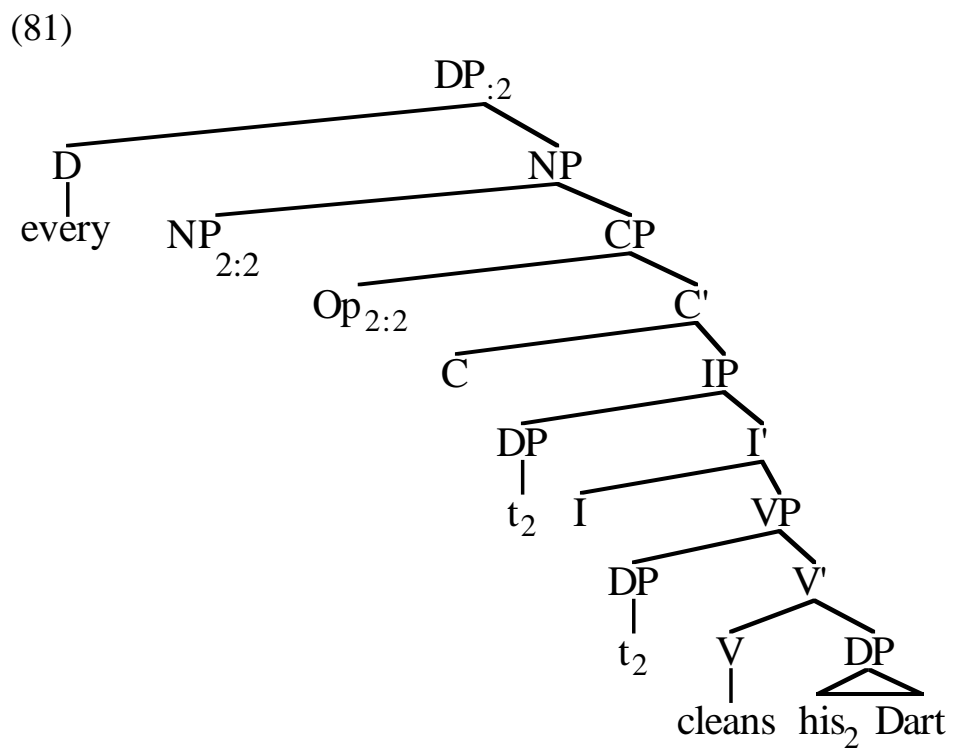

In his discussion of the relation between dependence and crossover effects, Higginbotham (1983:409) defines a formal variable as "an empty category that occurs in an argument position and is linked to a nonargument; the operator to which a formal variable is linked will be called its binder". I will assume that this definition is directly transposable into the Heim's model of anaphoric relations with the following modifications: a formal variable is an empty category that is linked to a c-commanding DP at LF. This modification reflects the fact that I assume QR to be optional, and ensures that if a trace in SpecVP is linked to a subject in SpecIP, it is a formal variable. In (81), both the trace of the relative operator in SpecIP and the internal subject trace in $\mathrm{SpecVP}$ are formal variables, as both are linked to the operator in SpecCP.

Higginbotham later notes that "the semantics of relatives justifies regarding [the trace of the moved subject and the trace of the relative operator] as distinct occurrences of the same formal variable." (1983:414) Applying this statement to the LF shown in (83), we can conclude that the VP internal traces (shown in boldface) are a "distinct occurrences of the same formal variable".

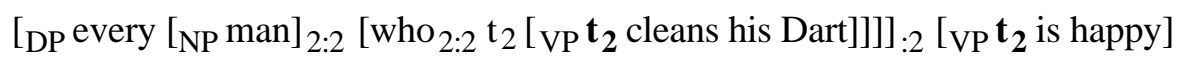

What is implicit in Higginbotham's analysis is that each distinct occurrence of the same formal variable is bound by a distinct occurrence of the same binder. In other words, the subject DP in (83) and the relative operator within this DP are distinct occurrences of the same binder, since the variables they bind are distinct occurrences of the same formal variable. ${ }^{23}$ The crucial point is that because the relative operator and the DP in an example like (83) can be considered distinct occurrences of the same binder, we may conclude that the distinct occurrences of a particular

23 The basic idea behind Higginbotham's analysis is that in the translation of a sentence like (83) into a logical representation, the relative clause is mapped into the restriction of the determiner every, while the VP is mapped into its nuclear scope (cf. Heim 1982, Diesing 1992). Empty categories bound by the DP in the matrix sentence and those bound by the relative operator in the relative clause are interpreted as cobound, i.e., bound by the determiner every.. A more intuitive, semantic representation of (83), where the variable $x$ has been substituted for the index 2 , is given in (i).

$$
\operatorname{every}_{x}(\operatorname{man}(\mathrm{x}) \& \operatorname{clean}(\mathrm{x}, \mathrm{x} \text { 's Dart) })(\operatorname{happy}(\mathrm{x}))
$$


formal variable in this and similar examples are cobound. This relation will play a crucial role in the analysis of Argument Contained Ellipsis to be presented in the next section.

\section{Argument Contained Ellipsis Reanalyzed}

\subsection{External Arguments}

The data presented in $\S 2.1$ were the basis for the generalization stated in (5), which is repeated below:

\section{Argument Contained Ellipsis}

Ellipsis between $\mathrm{VP}_{\alpha}$ and $\mathrm{VP}_{\beta}, \mathrm{VP}_{\beta}$ contained in an argument $\mathrm{A}_{\alpha}$ of $\mathrm{VP}_{\alpha}$, is licensed only if $A_{\alpha}$ is identical to the parallel argument $A_{\beta}$ of $\mathrm{VP}_{\beta}$.

(2a) and (2b), repeated below, exemplify this paradigm in the subcase of subject containment.

(2a) Every man who said he would buy some salmon did.

(2b) *Every man who said George would buy some salmon did.

(2b) is ungrammatical because the subjects of the related VPs are not identical. In the following paragraphs, I will show that the interaction of elliptical dependence, as implemented in $\S 3$, with referential dependence, as described in $\S 4$, forces argument identity in ACE structures.

In $\S 3$, I showed that the strict identity hypothesis requires the subjects of elliptically related VPs to bear identical outer indices. In the proposed model, the LFs of (2a) and (2b) are (84) and (85), respectively (omitting irrelevant details).

(84) [every man who ${ }_{1: 1} t_{1}$ said he ${ }_{1}$ would $\left[\operatorname{VP}^{t} t_{1} \text { buy some salmon] }\right]_{: 1}$ did [VP $\mathrm{t}_{1}$ buy some salmon]

[every man who ${ }_{1: 1} \mathrm{t}_{1}$ said George $3: 1$ would $\left[\mathrm{VP}_{\mathrm{t}} \mathrm{t}_{1} \text { buy some salmon] }\right]_{: 1}$ did $\left[\mathrm{VP}^{\mathrm{t}} \mathrm{t}_{1}\right.$ buy some salmon]

Both (84) and (85) appear to be well-formed LFs--in both examples, each argument is part of a chain that receives a theta role, satisfying Full Interpretation, and the VPs are strictly identical, in line with (50). What, then, is the crucial difference between (84) and (85) that makes (85) ungrammatical?

I claim that (85), but not (84), violates the LF well-formedness constraint (72) (*b-dependent $(\alpha, \alpha))$. According to the characterization of elliptical dependence that I have argued for in this paper, every syntactic entity in the LF representation of an elided VP is treated by the grammar as an occurrence of the syntactic entity that occupies the parallel position of an antecedent VP. The importance of this claim with respect to the data under discussion is that all VP internal traces in the LF of an elided VP are to be considered identical to their counterparts in the antecedent's LF. For example, in (84), repeated below, the boldfaced traces in the embedded and matrix VPs are to be considered as two occurrences of the same linguistic entity.

(84) [every man who 1:1 $_{1} \mathrm{t}_{1}$ said he ${ }_{1}$ would [ $\mathrm{VP}_{\mathbf{t}} \mathbf{t}_{\mathbf{1}}$ buy some salmon]]:1 did [VP $\mathbf{t}_{\mathbf{1}}$ buy some salmon]

The claim is that the relation between the traces in an elided VP and the traces in its antecedent is similar to but subtly different from the relation between the traces in the relative clause discussed in 
§5: whereas the latter are distinct occurrences of the same formal variable, I claim that the former are identical occurrences of the same formal variable.

With these assumptions in mind, we may now examine the b-dependence relations in the LFs (84) and (85). In both cases the trace in the specifier of the matrix VP is bound by the subject. By the definition of b-dependence, this trace is b-dependent on everything that is contained in the subject. It follows that the trace in the specifier of the matrix VP is dependent on the trace in the specifier of the embedded VP. According to the proposed characterization of elliptical dependence, these traces are identical, therefore the trace in the specifier of the matrix VP is b-dependent on itself, in violation of (72).

By this logic, however, both LFs should violate (72). In both cases, the trace in the matrix SpecVP is b-dependent on itself (the identical occurrence in the embedded SpecVP). To understand how identity of arguments ensures that (84) is grammatical despite its apparent illformedness with respect to (72), we must return again to the discussion of formal variables. Recall that in \$5 I suggested that if the trace of a moved DP with a relative clause modifier and the trace of the relative operator were to be considered distinct occurrences of the same formal variable, following Higginbotham (1983), then the binders of these variables should be considered distinct occurrences of the same binder, and the formal variables themselves should be considered to be cobound. This means that all occurrences of $\boldsymbol{t}_{1}$ in (84)--including the identical traces in the matrix and embedded SpecVP--are cobound. This is not true of (85), because the trace in the embedded SpecVP is within the scope of a closer potential binder, the DP George. In other words, the occurrences of the identical traces in (84) are cobound, but the occurrences of the identical traces in (85) are not cobound. This is the crucial difference between structures in which argument identity obtains and structures in which it does not. To understand how this difference ensures the wellformedness of examples manifesting argument identity, it is necessary to consider the basic idea behind b-dependence.

B-dependence states that " $\alpha$ ( $\alpha$ has no descriptive content) is b-dependent on $\beta$ if $\beta$ is contained in the binder of $\alpha$ ". The processes that generate LFs from S-structures, which are outlined in Heim (1992) and which I have assumed in this paper, create structures in which all potential syntactic binders (i.e., DPs with outer indices at S-structure) become operators at LF (cf. Higginbotham 1983). B-dependence is a formal statement of the relation between the variables that are bound in an operator's nuclear scope (by that operator) and the elements in the operator's restriction. It is the operator's restriction that determines the domain over which the variables in the nuclear scope may range; therefore, the bound variables in the nuclear scope are dependent for their interpretations on their binder's restriction. The restriction set must be established before the nuclear scope can be evaluated; similarly, it should never be necessary to know the value of some variable in the nuclear scope in order to establish the restriction. It follows that b-dependence must be irreflexive, and the data discussed in $\S 4.1$ support this conclusion.

The role of $b$-dependence in regulating the syntax-semantics interface can be most clearly stated in terms of indices (which, in Heim (1992), are what ultimately get translated as variables). B-dependence states that (the semantic translation of) any given LF must be such that the values of all indices contained in the binder of some index $i$ can be established before the value of $i$ itself is determined. Therefore, $i$ must not itself be contained in the binder of $i$, otherwise it would be necessary to know its value before its value could be determined, resulting in circularity. If it were the case, however, that $i$ occurred both in an operator's restriction and in its nuclear scope and the value of both occurrences of $i$ were set simultaneously, then, in effect, no b-dependence relation would hold between the two occurrences of $i$. In other words, if both occurrences of $i$ were cobound, it would not be the case that the value of one occurrence of $i$ would have to be ascertained in order to compute the value of the other occurrence of $i$. Neither would be dependent on the other. As noted above, this type of configuration is precisely what occurs in the LF of the 
grammatical sentence (84), but does not occur in the LF of the ungrammatical (85), both of which are repeated below.

[every man who ${ }_{1: 1} \mathrm{t}_{1}$ said he ${ }_{1}$ would $\left[\mathrm{VP}_{\mathrm{P}} \mathbf{t}_{\mathbf{1}} \text { buy some salmon] }\right]_{: 1}$ did [VP $\mathbf{t}_{\mathbf{1}}$ buy some salmon]

[every man who ${ }_{1: 1} \mathrm{t}_{1}$ said George $3: 1$ would $\left[{ }_{V P} \mathbf{t}_{\mathbf{1}} \text { buy some salmon] }\right]_{: 1} \operatorname{did}\left[{ }_{V P} \mathbf{t}_{\mathbf{1}}\right.$ buy some salmon]

In each example, the relevant traces are in boldface. The crucial difference between the two structures is that in (84), the trace in the matrix SpecVP is bound by the QRed DP and the trace in the embedded SpecVP is bound by the relative operator, therefore both occurrences of $t_{1}$ are bound by (distinct occurrences of) the same binder--they are cobound. In (85), however, $\boldsymbol{t}_{1}$ in the matrix SpecVP is bound by the subject of the matrix clause, while $t_{1}$ in the embedded SpecVP is bound by the DP George; these DPs are different binders. Because the identical occurrences of $\boldsymbol{t}_{\boldsymbol{1}}$ in the restriction and nuclear scope in (72) are cobound, no b-dependence relation holds between them. If no b-dependence relation holds between the traces, then (84) is vacuously well-formed with respect to the LF constraint (72). (85), in which there is a b-dependence relation between the identical traces, violates (72). of (85).

The analysis of Wasow's sentence (1), repeated below, is exactly the same as the analysis

(1) *A proof that God exists does.

According to the strict identity hypothesis, the LF of (1) must be as in (86):

$$
\text { [a proof that } \operatorname{God}_{2: 1}\left[\mathrm{vP}_{\mathbf{1}} \mathbf{t}_{\mathbf{1}} \text { exists] }\right]_{3: 1} \text { does }\left[\mathrm{vP} \mathbf{t}_{\mathbf{1}} \text { exist }\right]
$$

(86) is ill-formed for the same reason as (85): the trace in the matrix VP is b-dependent on an identical occurrence of that trace in the subject-contained VP, in violation of (72). The difference between Wasow's example and e.g. (85) is that for (1), there is no corresponding grammatical sentence in which subject identity holds. The explanation of this fact parallels the discussion of $\mathrm{i}$ within-i violations in $\$ 4.1$ (cf. exx. 73-77). In sentences like (2a-b), the argument-contained VP is inside a relative clause modifier of the subject. Binding of the subject of this VP by the relative operator prevents a reflexive b-dependence relation from obtaining within the subject DP. In (1), however, the argument-contained VP is in a selected complement of $\mathrm{N}^{0}$. In order for the reflexive b-dependence relation between the VP internal traces to be eliminated, the subject of the contained VP must itself be bound by the subject of the matrix VP. This configuration would be ill-formed with respect to (72), however, because the embedded subject would be contained in its binder, the matrix subject. This structure is shown in (87).

$$
\text { [a proof that it }{ }_{1}\left[\mathrm{VP} \mathbf{t}_{\mathbf{1}} \text { is correct] }\right]_{2: 1}\left[\mathrm{VP} \mathbf{t}_{\mathbf{1}}\right. \text { is correct] }
$$

Although the b-dependence relation between the identical traces has been eliminated, a new one has been created: the subject of the embedded VP $i_{1}$ is contained in its binder, the subject of the matrix VP. Therefore $i t_{1}$ is b-dependent on itself, in violation of (72).

\subsection{Internal Arguments}

The other cases of Argument Contained Ellipsis can be analyzed in exactly the same manner as the subject cases have been. The other configurations discussed were examples of 
antecedent contained deletion structures. Examples like (3a-b) involve sentences in which one of the VPs is contained in an internal argument of the other; examples like (88-89) show sentences in which one of the elliptically related VPs is contained in the external argument of an embedded VP (cf. §2.1.2).

(3a) Polly visited every town Eric did.

(3b) *Polly visited every town in every country Eric did.

(88) Sydney believes every proposal Evelyn does to be insane.

(89) *Sydney believes every aspect of every proposal Evelyn does to be insane.

Following May (1985), I assume that in order for an ACD structure to be interpreted, the phrase containing the elided VP must undergo QR at LF (in order to eliminate the antecedent containment configuration). The LFs corresponding to (3a-b) and (88-89) are given in (90-91) and (92-93), respectively. 24

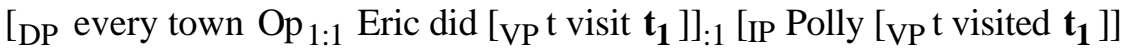

$$
\begin{aligned}
& {\left[\mathrm{DP} \text { every town in [DP } \text { every country } \mathrm{Op}_{1: 1} \text { Eric did }\left[\mathrm{VP}_{\mathrm{V}} \mathbf{t} \text { visit } \mathbf{t}_{\mathbf{1}}\right]_{: 1}\right]_{: 1}}
\end{aligned}
$$

[DP every proposal Op ${ }_{1: 1}$ that Evelyn does [ $\mathrm{VP}_{\mathrm{D}} \mathrm{t}$ believe [IP $\mathbf{t}_{\mathbf{1}}$ to be insane]]: 1 [Sydney [VP $\mathbf{t}$ believes [IP $\mathbf{t}_{\mathbf{1}}$ to be insane]]]

[DP every aspect of [ ${ }_{\mathrm{DP}}$ every proposal Op $\mathrm{p}_{1: 1}$ that Evelyn does [ $\mathrm{VP}_{\mathrm{T}} \mathrm{t}$ believe [IP $\mathbf{t}_{\mathbf{1}}$ to be insane] $\left.]_{: 1}\right]_{: 1}\left[\right.$ Sydney [ $\mathrm{VP}$ t believes [IP $\mathbf{t}_{\mathbf{1}}$ to be insane]]]

In each example, the DP containing the surface null VP has been QRed, leaving a trace which is contained in the antecedent VP. The resulting LFs are the structures with respect to which the identity relation described in (50) must hold. These must be evaluated for a potential b-dependence relation between the trace in the LF representation of the elided VP and the trace in the antecedent VP. These examples contain exactly the same configuration as the examples of subject containment discussed above: a trace in the matrix VP is b-dependent on a trace in a VP contained in a relative clause. According to the strict identity hypothesis, these traces are identical, so these structures should violate (72).

The explanation of the contrast between the examples in which argument identity holds and those in which it does not parallels the explanation of the subject identity examples. In the grammatical sentences (90) and (92), the relative operator is linked to the DP that has undergone $\mathrm{QR}$, therefore, according to the assumptions made above, both occurrences of the identical trace (underlined) are bound by distinct occurrences of the same binder. As in the subject examples discussed above, if the identical traces are cobound, no b-dependence relation holds between them. The LF constraint (72) does not apply to (90) and (92).

In (91) and (93), however, the relative operator is not linked to the DP that has undergone $\mathrm{QR}$, but to a different DP contained within the QRed argument. In these cases, it cannot be said that the relative operator and the QRed DP are distinct occurrences of the same binder; they are

\footnotetext{
${ }^{24}$ For clarity, I will only show indexing on the relevant DPs (the ones involved in the argument containment configuration). Although the subjects of the matrix VPs will necessarily bear the same outer indices (because the matrix VP is what is copied), this index does not play a role in the bdependence relation involved in the ungrammatical sentences.
} 
completely different binders. The identical traces in these examples are not cobound, therefore the trace in the antecedent VP is b-dependent on the trace within the elided VP. Because these traces are identical, (91) and (93) violate (72).

\subsection{Summary}

To summarize, it has been shown that structural configurations in which one of two elliptically related VPs is contained in an argument of the other (what I have referred to as Argument Contained Ellipsis) violate the LF well-formedness constraint $* b$-dependent $(\alpha, \alpha)$. This constraint regulates the syntax-semantics interface by ruling out circular constructions on the basis of syntactic indexing configurations. If, as I have argued, variables (inner indices on traces) in an elided VP's LF are identical to the parallel variables in an antecedent VPs LF, then Argument Contained Ellipsis configurations will inevitably violate this constraint, because one of the identical variables will be contained in the binder of the other. This relation is eliminated, however, just in case both occurrences of the identical variable are cobound. Basing my argument on Higginbotham's (1983) discussion of relative clauses, I showed that a cobinding relation will hold when the parallel arguments of the related VPs are identical. If the b-dependence relation between the identical variables is eliminated, then the structure will vacuously satisfy *b-dependent $(\alpha, \alpha)$. The interaction of the revised theory of referential dependence presented in $\$ 4$ and a theory of elliptical dependence, as implemented by the strict identity hypothesis, thus forces argument identity in cases of Argument Contained Ellipsis.

\section{Elliptical Dependence and Alphabetic Variance}

Two basic claims of this paper are that (72) reflects a general semantic constraint against referential circularity and that sentences that contain indexing configurations that would lead to a violation of this constraint are ruled out at LF. Argument Contained Ellipsis structures in which the argument identity constraint described in (5) does not hold have been argued to contain such illformed LFs. The analysis of ACE presented in this paper has implications beyond the explanation of a puzzling set of data, however. In particular, it may provide a principled explanation of a wellestablished but nevertheless stipulative constraint on VP identity in elliptical structures.

The basic structure of the LF of an example of ACE is shown in (94), where the occurrences of $e_{1}$ are identical:

$$
\left[\mathrm{DP} \ldots\left[\mathrm{VP}_{1} \ldots \mathrm{e}_{1} \ldots\right] \ldots\right]_{: 1}\left[\ldots\left[\mathrm{VPP}_{2} \ldots \mathrm{e}_{1} \ldots\right] \ldots\right]
$$

(94) is the LF syntactic representation of a tripartite quantification structure in the semantic component (cf. §5, fn.27). The hypothesis advanced in this paper is that an elliptical relation between $\mathrm{VP}_{1}$ and $\mathrm{VP}_{2}$ is licensed in these configurations only if the identical occurrences of the $\mathrm{VP}$ internal variable are cobound; otherwise the LF is ruled out by (72) as a circular structure.

This constraint has a precedent in the literature on VP ellipsis. In Sag (1976), two VPs may stand in an elliptical relation iff the lambda-expressions that correspond to their logical forms are alphabetic variants. There are conditions on this relation, though, one of which is stated in (95) (Sag 1976:104).

(95) For two $\lambda$-expressions $\lambda x$.A and $\lambda y$.B to be alphabetic variants, if some operator $\varphi$ outside $\lambda x$.A binds a variable in $A$, then the corresponding variable in $\lambda$ y.B must also be bound by $\varphi$.

Arguably, (94)--and therefore all cases of ACE--is a configuration that fits the structural description in (95). The VPs correspond to $\lambda \mathrm{x}$.A and $\lambda \mathrm{y} . \mathrm{B}$, the VP internal traces correspond to 
variables within the lambda expressions, and DP corresponds to $\varphi$. Therefore, according to (95), if the trace in the matrix VP is bound by DP, the trace in the embedded VP must also be bound by DP. This will be true just in case argument identity holds (cf. the discussion of relative clauses in $\S 5)$.

If ACE structures are construed in this way, then in a model of ellipsis along the lines of Sag (1976), the argument identity constraint follows directly from (95). In structures in which argument identity holds the related VPs are alphabetic variants; in structures in which argument identity does not hold they are not alphabetic variants.

Although (95) gets the right results, it is a stipulation. This constraint states that in configurations such as (94), the variables in the related VPs must be cobound. It does not, however, explain why. Moreover, there is reason to believe that this constraint is too strong. (95) correctly rules out ellipsis in the second sentence in (96):

(96) Who did Martin see? *Who did Bill?

It is not clear that (95) is the correct explanation of such sentences, however. ${ }^{25}$ Jacobson (1992) presents a number of sentences that are problematic for the alphabetic variance approach (see also exx. 48-49, §2.2.3; examples 98-99 are from Evans 1988):

(97) I know which student Al likes, and I know which student Mary doesn't.

(98) Bagels, I like. Donuts, I don't.

(99) John was supposed to read several books this semester. But the only one that he actually did was The Brothers Karamazov.

According to (95), each of these examples should be ungrammatical because each sentence contains variables in elliptically related VPs that are bound by distinct operators. This is clearly the wrong result.

The important question raised by examples like (97-99) is whether (95) is active only in syntactic configurations that correspond to tripartite structures in the semantics. If so, then (95) takes on the status of (5), the original description of the conditions licensing Argument Contained Ellipsis. As with (5), we have the intuition that if (95) is true only of certain configurations, it must reflect some deeper fact about ellipsis. If the analysis of ACE can be generalized to all tripartite quantification structures (where one VP is in the restriction and the other in the nuclear scope), then the analysis developed in this paper claims that the fact reflected by (95) is referential circularity. (95) need not be stated, except as a descriptive law of VP ellipsis, because the requirement that the variables in the VPs in structures like (94) must be cobound follows from the interaction of elliptical dependence and a very general claim about the syntax-semantics interface: the binding relations represented in the syntax by indexing configurations must be such that they do not give rise to referential circularity in the semantics.

\section{Concluding Remarks}

This paper has argued that a straightforward account of the argument identity constraint that holds in Argument Contained Ellipsis structures follows from the interaction of two types of dependence relations: elliptical dependence, which characterizes the relation between an elided VP

\footnotetext{
25 The analysis proposed in this paper has no account of the ungrammaticality of (96) in terms of ill-formed indexing configurations (and would, in fact, predict it to be grammatical). Given the grammaticality of examples like (97-99), I will assume that an explanation of of (96) will be found outside of conditions on indexing configurations, and will leave a resolution of this issue for future work.
} 
and the VP from which it receives its interpretation, and referential dependence, which characterizes the relation between a nominal anaphoric element and its antecedent. Several important results came out of the discussion.

First, the paper supports the theory of anaphoric relations developed in Heim (1992). Heim (1992:3) notes that her revision of Reinhart (1983) may share with Reinhart the "wrong descriptive generalizations" about ellipsis. Although I have avoided discussion of some of the problems associated with syntactic accounts of ellipsis (several of which are discussed in Wescoat 1989 and Dalrymple 1991), I have shown that Heim's system allows us to implement an explicit representation of elliptical dependence via the strict identity hypothesis, and thereby give a precise account of a complex array of facts.

Second, this work relativizes the concept of referential dependence developed in Higginbotham (1983) and Haïk (1987) to a system of anaphoric relations in which binding and coreference are represented separately. This relativization of dependence was achieved by dividing it into the $r$-dependence and b-dependence, which are active in coreference and binding relations, respectively. The formulation of b-dependence as a distinct dependence relation provided a direct explanation of the grammaticality of certain types of apparent $i$-within-i violations, which were previously accounted for stipulatively, and was of crucial importance to the analysis of ACE. Future work should be directed towards exploring these relations (e.g., with respect to Bach-Peters sentences). In particular, it should be ascertained at what level, if at all, $r$-dependence and $b$ dependence interact.

Finally, this work makes a fundamental claim about ellipsis, namely that the relation between an elided VP and the VP from which it receives its interpretation, which I have referred to as elliptical dependence, should be directly encoded in a theory of ellipsis. I suggested that this relation can be implemented in a syntactic model of ellipsis by the strict identity hypothesis, which requires elliptically related VPs to be identical in terms of indexical or variable value at Logical Form, then showed that the interaction of elliptical dependence and referential dependence accounts for the Argument Contained Ellipsis facts.

Appendix A: Kitagawa (1991)

Kitagawa proposes that VP ellipsis is interpreted by copying the LF syntactic structure of an antecedent VP into the position of a null VP by an operation of "VP Copy". The post-Copy LF is then subject to various grammatical constraints, including the binding theory, which he assumes to work in the following way (Kitagawa 1991:501):

(A1) A-binding at $L F$

All A-binding and its concomitant coindexation (including that for NP-traces) takes place in the LF component.

(A2) Binding Theory $A / B / C$ at $L F$

The principles $\mathrm{A} / \mathrm{B} / \mathrm{C}$ of the $\mathrm{BT}$ must be satisfied in the $\mathrm{LF}$ component.

Kitagawa does not assume extrinsic ordering of (A1) (the assignment of indices to A-bound DPs) and VP Copy; however, if assignment of indices occurs before VP Copy, (A2) will rule out the ensuing reconstruction as a Principle A violation. This process is illustrated by the following pair of examples.

(A3) Sartre $_{1}\left[\mathrm{vP}_{1}\right.$ scratched himself $\left._{1}\right]$ and Camus ${ }_{2}$ did [ $\mathrm{vP}_{2} \mathrm{t}_{2}$ scratch himself $\left.{ }_{2}\right]$ too (A4) $*$ Sartre $_{1}\left[\mathrm{vPt}_{1}\right.$ scratched himself $\left._{1}\right]$ and Camus ${ }_{2}$ did $\left[\mathrm{vP}_{1} \mathrm{t}_{1}\right.$ scratch himself $\left.{ }_{1}\right]$ too 
Indexing prior to VP Copy creates the illegal structure (A4), whereas post-Copy indexing in (A3) has the desired effect of indexing the internal subject trace with the DP in SpecIP. ${ }^{26}$

Let us see how this model fares with respect to the ACE data. I will limit my discussion to the subject cases, as these are the examples that Kitagawas's analysis cannot account for. The VPinternal subject trace in SpecVP is A-bound; according to the assumptions stated above, then, it may receive an index after VP Copy has applied. (In (A4) above, indexing must be allowed to follow VP Copy.) This analysis predicts that the LF the ungrammatical example (2b) (repeated below), will be (A5), which is well-formed and should therefore be grammatical.

(2b) *Every man who said George should buy some salmon did.

(A5) [DP Every man who said George ${ }_{2}$ should $\left[{ }_{V P} t_{2} \text { buy some salmon] }\right]_{1}$ did [VP $\mathrm{t}_{1}$ buy some salmon]

The analysis of Wasow's original example (1) is the same: because A-bound traces may be indexed after VP Copy has applied, (A6), which is well-formed, is a potential LF of (1).

(1) *A proof that God exists does.

(52) [DP A proof that $\operatorname{God}_{2}\left[\mathrm{VP}_{2} \mathrm{t}_{2}\right.$ exists $\left.]\right]_{1}$ does $\left[\mathrm{VP}_{1} \mathrm{t}_{1}\right.$ exist $]$

(1) is incorrectly predicted to be grammatical, suggesting that a unified explanation of the ACE facts must be sought elsewhere.

Appendix B: Fiengo \& May (1993)

Fiengo and May (FM) (1993:310) characterize ellipsis as "the absence of lexical projection"; it is syntactic structure that has not been licensed lexically, that cannot be licensed until certain identity conditions are met. These conditions can be met at LF when the non-projected material is reconstructed. In FM's model, reconstruction is an identity relation over phrase markers. $\varphi$ is a reconstruction of $\psi$ iff $\varphi$ and $\psi$ share "syntactic identity, up to vehicle change and indexical variation under Dependency Theory." (FM 1993:248) Syntactic identity preserves "grammatical category, as well as linear, domination and government relations; all occurrences will be structurally composed in exactly the same way." (1993:247) Vehicle change is a process that changes the value of the nominal feature [+/-pronoun] under certain circumstances; as this process is irrelevant to the current discussion, I will not discuss it further. The particular aspect of this definition of reconstruction that is important to the analysis of the data presented in $\$ 2.1$ is the fact that a reconstruction $\mathrm{R}$ of some phrase-marker $\mathrm{P}$ allows "indexical variation under Dependency Theory"; that is, under the appropriate circumstances, the indexical values of DPs in a phrase marker $\mathrm{P}_{1}$ may differ from the indexical values of DPs in a phrase marker $\mathrm{P}_{2}$, yet $\mathrm{P}_{1}$ and $\mathrm{P}_{2}$ may still stand in the reconstruction relation. It is in this way that FM's model of ellipsis requires loose rather than strict identity of elliptically related VPs To understand when indexical variation among reconstructions is permitted, we must take a brief look at Dependency Theory.

Dependency Theory, which governs referential relations between DPs, is parallel to binding theory, which governs indexical relations between DPs. Moreover, Dependency Theory provides additional information about the type of index assigned to a given DP. Dependency Theory defines two types of indices: $\alpha$-occurrences, which are assigned reference independently of other linguistic elements; and $\beta$-occurrences, which must receive their referential value from some

26 Obligatory sloppy readings of reflexive pronouns are accounted for similarly. I refer the interested reader to Kitagawa (1991) for a more detailed presentation of how this theory accounts for the distribution of strict and sloppy identity readings in elliptical structures. 
anteceding linguistic element (FM 1993:52). The effect of these definitions is that only $\beta$ occurrences play a role in dependencies, as only $\beta$-occurrences have no independent referential value. An indexical dependency is the relation between a (sequence of) $\beta$-occurrence(s) of an index $i$ and its antecedent, an $\alpha$-occurrence. This relation can be schematically represented as a triple $\left\langle\left(c_{1}{ }^{\alpha}, c_{2} \beta, \ldots, c_{n} \beta\right), I, S D>\right.$, where $\left(c_{1}^{\alpha}, c_{2} \beta, \ldots, c_{n} \beta\right)$ is a sequence of elements bearing the index I, I is an integer, and SD is a structural description specifying the structure which connects the bearers of the index I. (FM 1993:56) For example, the indexical dependency holding between the subject and the pronoun in (B1) can be schematically represented as in (B2).

(B1) Hector $_{1}{ }^{\alpha}$ loves his $_{1}{ }^{\beta}$ Dart.

(B2) $\left\langle\left([\text { Hector }]^{\alpha},[\text { his }]^{\beta}\right), 1,\langle\mathrm{DP}, \mathrm{V}, \mathrm{DP}>>\right.$

The question that we must ask with respect to reconstruction is the following: when does Dependency Theory define two indexical dependencies as identical? FM answer this question in the following way (FM 1993:100):

(B3) Indexical dependencies ID and ID' are i-copies iff ID and ID' vary from each other in at most the value of I.

In other words, indexical dependencies are identical if and only if the $\beta$-occurrences in each ID depend on elements which are "structurally parallel". The sense in which FM's statement of reconstruction involves loose identity should now be clear: if $\varphi$ and $\psi$ stand in the reconstruction relation, then indices on parallel elements in $\varphi$ and $\psi$ are either identical or part of identical indexical dependencies (i-copies). It should be noted that because we are defining the conditions under which distinct numerical indices can be construed as identical in terms of indexical dependencies, and only $\beta$-occurrences are involved in indexical dependencies, only indexical variation of $\beta$ occurrences will be permitted in a legal reconstruction; the numerical value of all $\alpha$-occurrences must be the same across all phrase markers that stand in the reconstruction relation.

Indexical variation under Dependency Theory ensures that the indexical value of the reconstructed subject trace in SpecVP may vary in order to satisfy the well-formedness constraints imposed upon it. Consider the following S-structure:

$$
\operatorname{Martin}_{1}{ }^{\alpha}\left[\mathrm{VP}_{1} \mathrm{t}_{1}^{\beta} \text { left }\right] \text { and } \mathrm{Bill}_{2}{ }^{\alpha} \text { did too. }
$$

For the second conjunct to be interpreted, a VP must be reconstructed in which SpecVP is occupied by a trace bearing a $\beta$-occurrence of the index 2 , as shown in (B5).

$$
\operatorname{Martin}_{1}{ }^{\alpha}\left[\mathrm{VP}_{1} \mathrm{t}_{1}^{\beta} \text { left }\right] \text { and Bill }{ }_{2}^{\alpha} \operatorname{did}\left[\mathrm{VP}_{2}{ }^{\beta} \text { leave }\right] \text { too }
$$

In order to decide whether this VP is a legal reconstruction, we must check to see whether the traces in the two VPs are part of identical indexical dependencies. The indexical dependencies in the first and second conjuncts of (B5) are given in (B6) and (B7).

$$
\begin{aligned}
& <\left([\text { Martin }]^{\alpha},[\mathrm{t}]^{\beta}\right), 1,\langle\mathrm{DP}, \mathrm{DP}, \mathrm{V}\rangle> \\
& <\left([\mathrm{Bill}]^{\alpha},[\mathrm{t}]^{\beta}\right), 2,\langle\mathrm{DP}, \mathrm{DP}, \mathrm{V}>>
\end{aligned}
$$

(B6) and (B7) are identical except for indexical value, so they are i-copies, and (B5) contains a legal reconstruction.

We may now turn to an analysis of Argument Contained Ellipsis. Does FM's system explain why the descriptive generalization stated in (5) should hold? Again, I will only concentrate 
on cases of subject containment. Like Kitagawa (1991), FM's system is ultimately unsuccessful in ensuring argument identity in the case of subjects. Consider (2a-b), repeated below.

(2a) Every man who said he would buy some salmon did.

(2b) *Every man who said George should buy some salmon did.

Our concern is whether (2b) is predicted to be ungrammatical. ${ }^{27}$ FM's definition of reconstruction permits two possible post-reconstruction LFs for (2b), which are shown in (B8) and (B9).

(B8) $\quad\left[\right.$ DP every man who said George ${ }_{1}^{\alpha}$ would $\left[\mathrm{vP}_{1}{ }^{\beta} \text { buy some salmon }\right]_{2}{ }^{\alpha} \mathrm{did}$ [VP $\mathrm{t}_{1} \beta$ buy some salmon]

(B9) $\quad\left[\text { DP every man who said George }{ }_{1}^{\alpha} \text { should }\left[\mathrm{vPt}_{1}{ }^{\beta} \text { buy some salmon }\right]\right]_{2}{ }^{\alpha}$ did [VPt $\mathrm{t}_{2} \beta$ buy some salmon]

In (B8), the reconstructed VP is identical to the source VP in every respect. This structure is illformed, however, because the matrix subject is not coindexed with the trace in the specifier of the reconstructed VP. (B9), however, is a well-formed LF. Moreover, the reconstructed VP in (B9) is a legal reconstruction, because the indexical dependency that holds between the matrix subject and the trace in the reconstructed SpecVP is an i-copy of the indexical dependency that holds between the embedded subject George and the trace in the embedded SpecVP. The two indexical dependencies differ only in indexical value, as shown in (B10).

(B10) $<\left([\text { every man.... }]^{\alpha},[\mathrm{t}]^{\beta}\right), 2,\langle\mathrm{DP}, \mathrm{DP}, \mathrm{V}\rangle>$ $\left\langle\left([\text { George }]^{\alpha},[\mathrm{t}]^{\beta}\right), 1,\langle\mathrm{DP}, \mathrm{DP}, \mathrm{V}\rangle\right\rangle$

Thus FMs system predicts the ungrammatical sentence ( $2 b)$ to be grammatical, because there is a legal reconstruction of the elided VP.

The analysis of Wasow's example (1) is exactly parallel to the analysis of (2a). In order for (1) to be well-formed, it would have to have an LF in which the VP internal subject trace in the reconstructed VP is coindexed with the matrix subject, as in (B11).

(1) *A proof that God exists does.

(B11) [DP A proof that $\operatorname{God}_{1} \alpha\left[\mathrm{VP}_{1}{ }_{1} \beta \text { exists }\right]_{2}{ }^{\alpha}$ does $\left[\mathrm{VP}_{2} \mathrm{t}_{2} \beta\right.$ exist $]$

As was the case for (B10), the indexical dependencies in the related VPs in (B11) differ only in indexical value, as shown in (B12).

(B12) <([a proof that... $\left.]^{\alpha},[\mathrm{t}]^{\beta}\right), 2,\langle\mathrm{DP}, \mathrm{DP}, \mathrm{V}>>$ $<\left([\mathrm{God}]^{\alpha},[\mathrm{t}]^{\beta}\right), 1,\langle\mathrm{DP}, \mathrm{DP}, \mathrm{V}>>$

Since the indexical dependencies in the elliptical related VPs are i-copies, the LF of the elided VP in (B11) is a legal reconstruction of the antecedent VP embedded in the subject, and (1) is predicted to be grammatical.

${ }^{27}$ The grammaticality of (2a) is straightforwardly accounted for. Because the subjects of the related VPs will be coindexed, the reconstructed VP will have exactly the same form as the antecedent VP. 
Appendix C: The Strict Identity Hypothesis

\author{
C.1 Strict and Sloppy Ambiguity \\ Consider (C1):
}

(C1) Eugene promoted his kvass and Sasha did too.

(C1) has two readings: one in which Sasha promoted his own kvass (the sloppy reading), and one in which Sasha promoted Eugene's kvass (the strict reading). The explanation of these facts in the current system is essentially that of Reinhart (1983). Because the subjects of the related VPs must bear identical outer indices, any pronoun in the sentence containing antecedent VP that is bound by the subject (and therefore linked to it) must be bound by the subject in the sentence containing the elided VP in order for strict identity to obtain. This configuration, shown in (C2), corresponds to the sloppy identity reading of $(\mathrm{C} 2)$.

(C2) Eugene ${ }_{2: 1}\left[\mathrm{VP}^{\mathrm{t}_{1}}\right.$ promoted his $_{1}$ kvass $]$ and $\mathrm{Sasha}_{3: 1}$ did $\left[\mathrm{VP}_{\mathrm{P}} \mathrm{t}_{1}\right.$ promote his $_{1}$ kvass] too.

If, on the other hand, the pronoun in the first conjunct is interpreted as coreferential with the subject, and therefore colinked with but not linked to it at LF, the pronoun in the LF representation of the elided VP will not be linked to the subject in the second conjunct. As identity of inner indices entails coreference (cf. §3.3), the pronoun in the elided VP's LF will receive the same interpretation as the pronoun in the antecedent VP's LF: its reference in both cases will be the A-subject. This "strict" interpretation of (C1) corresponds to the LF shown in (C3):

(C3) Eugene ${ }_{2: 1}\left[{ }_{V P} t_{1}\right.$ promoted his ${ }_{2}$ kvass] and Sasha ${ }_{3: 1}$ did $\left[{ }_{V P} t_{1}\right.$ promote his $_{2}$ kvass] too.

Because indexing at S-structure is free and optional, both (C2) and (C3) are potential LFs of $(\mathrm{C} 1)$. The strict/sloppy ambiguity associated with sentences like (C1) is thus expected if we assume the binding theory of Heim (1992) and a strict identity model of ellipsis.

C.2 Ellipsis in Non-conjoined Structures

An example of antecedent contained deletion (ACD) is illustrated by $(\mathrm{C} 4)$ :

(C4) Ivan loves every woman who Mitya does.

Following May (1985), I will assume that ACD structures may be interpreted only if the complement of the matrix verb has undergone $\mathrm{QR}$ at $\mathrm{LF}$ in order to eliminate the antecedent containment configuration. According to (50), the LF of (C4) must be (C5):

(C5) [every woman $\left[\text { who }_{6: 6}\left[\text { Mitya }_{3: 1} \text { does }\left[\mathrm{VP}_{1} \mathrm{t}_{1} \text { love }_{6}\right]\right]\right]_{: 6}\left[\operatorname{Ivan}_{2: 1}\left[\mathrm{VP} \mathrm{t}_{1}\right.\right.$ loves $\left.\left.\left.\mathrm{t}_{6}\right]\right]\right]$

The primary concern is, of course, whether the S-structure corresponding to (C5) (i.e., C4) involves a Condition C violation. Given Heim's (1992) definitions of indexing configurations, there is no reason to believe that the structural representation in $(\mathrm{C} 4)$ should be ruled out. By defining binding in terms of linking, we have ensured that the c-commanded r-expression in $(\mathrm{C} 4)$ (the subject of the embedded VP) is free: its inner index is not equal to the outer index of the 
subject of the matrix VP. Equality of outer indices will never cause two DPs to be in a binding relation.

The analysis of ACD is straightforward; constructions that seem more problematic at first glance are those similar to (C6), in which the pronominal subject of the null VP is deictic, that is, not interpreted as a variable bound by the subject of the antecedent VP.

(C6) Every poet composed his rhymes in just the way he did.

If the pronominal subject of the elided VP is deictic, then it must not be bound by the quantified DP every poet. If the manner phrase which contains the null VP is adjoined to VP, however, the quantified DP c-commands the pronoun. In a single indexing system, the requirement that the subjects of elliptically related VPs be coindexed would force binding of the pronoun by the QDP. In the double indexing system of Heim (1992), however, the reading in which the pronoun is deictic is derived by allowing the pronoun and the QDP to bear distinct inner indices; the fact that their outer indices are the same is irrelevant to binding conditions. ${ }^{28}$ The LF of (C6) with this construal is given in (C7):

(C7) Every poet:1 $\left[_{V P}\left[{ }_{V P} t_{1}\right.\right.$ composed his ${ }_{1}$ rhymes] [PP in just the way he ${ }_{3: 1} \operatorname{did}_{V_{P P}} t_{1}$ composed his ${ }_{1}$ rhymes]]

Binding is defined in terms of linking; because the pronoun is not linked to the QDP, it is not bound by the QDP. If the strict identity model of ellipsis is accepted, then (C7) supports Heim's claim that Reinhart's original indexing system must be elaborated. In Reinhart's system, the pronoun would be coindexed with and c-commanded by the QDP, hence obligatorily construed as bound by the QDP. The reading expressed by (C7) would be unavailable.

Note that the indexing in (C7) forces a sloppy reading of the genitive pronoun his: because the occurrence of this pronoun in the elided VP is linked to and c-commanded by the subject of the elided VP, it is bound by this DP, forcing the sloppy reading. This seems to be the most salient reading of (C7), but as pointed out to me by Mary Dalrymple (personal communication), a structurally similar sentence, such as (C8), allows a strict reading.

(C8) Every student read his paper before the teacher did.

(C8) has two readings: one in which the teacher read his own paper (the sloppy reading), and one in which the teacher read every student's paper (the strict reading). The LF corresponding to the first reading is parallel to (C7):

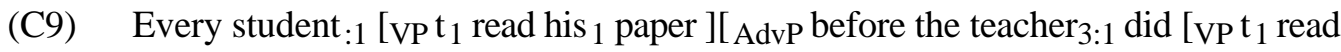
his 1 paper]]]

According to the strict identity hypothesis, the DP the teacher must bear the same outer index as the subject of the antecedent VP every student. If (C9) were the only possible LF of (C8), then, we would have no explanation for the fact that $(\mathrm{C} 8)$ has a strict interpretation. Given our assumptions about indexing and $\mathrm{QR}$, however, it is not the case that (C9) is the only possible LF of this sentence. Recall that both outer indexing and QR are assumed to be optional, but a DP must bear an outer index if it does undergo QR (in order to bind a variable in a theta position at LF). There is

\footnotetext{
${ }^{28}$ Of course, a pronoun that bears an outer index is interpreted as an operator at LF like any other DP that bears an outer index. Similar cases involving bound variable prounouns are discussed in Heim (1992).
} 
an LF of (C8), then, in which the DP his paper has undergone QR. If this DP has undergone QR in the antecedent clause, then, in order for strict identity to obtain, the LF of the elided VP must contain a trace as the complement of $\mathrm{V}$ that bears an inner index that is identical to the outer index of the moved DP. The moved DP will bind both the trace in the antecedent VP and the trace in the elided VP, ensuring the proper interpretation. This LF is shown in (C10): 29

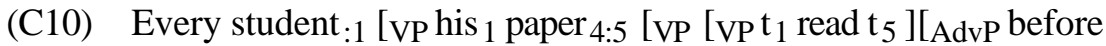
[IP the teacher $3: 1$ did $\left.\left.\left.\left.\left[\mathrm{VP}_{1} \mathrm{read}_{5}\right]\right]\right]\right]\right]$

In $(\mathrm{C} 10)$, although the teacher bears the same outer index as the pronoun his, it does not ccommand this pronoun, and so does not bind it. The DP every student does, however, c-command and therefore bind this pronoun, ensuring the strict reading.

29 Since VP is a clausal node, the moved DP may adjoin to it, ensuring that the pronoun his is bound by the subject at LF. 
References

Baltin, Mark. 1993. VP-Ellipsis as Non-Lambda Predicate Ellipsis. Ms., New York University. Chao, Wynn. 1988. On Ellipsis. Garland: New York.

Chomsky, Noam. 1986a. Barriers, Cambridge: MIT Press.

Chomsky, Noam. 1986b. Knowledge of Language: Its Nature, Origin, and Use, New York: Praeger.

Chomsky, Noam. 1981. Lectures on Government and Binding. Dordrecht: Foris.

Chomksy, Noam. 1957. Syntactic Structures. The Hague: Mouton.

Chung, Sandra, Jim McCloskey, and William Ladusaw. 1992. Sluicing and Logical Form. Ms., University of California, Santa Cruz.

Dalrymple, Mary. 1991. Against Reconstruction in Ellipsis. Ms., Xerox-PARC and Center for the Study of Language and Information.

Dalrymple, Mary, Stuart Shieber, and Fernando Pereira. 1991. Ellipsis and Higher Order Unification. Linguistics and Philosophy 14:399-452.

Diesing, Molly. 1992. Indefinites. Cambridge: MIT Press.

Evans, Frederic. 1988. Binding into Anaphoric Verb Phrases. In Proceedings of the 5th Annual Eastern States Conference on Linguistics, ed. J. Powers and K. de Jong, 122-129.

Evans, Gareth. 1977. Pronouns, Quantifiers, and Relative Clauses. Canadian Journal of Philosophy 7.3:467-537.

Fiengo, Robert and Robert May. 1990. Anaphora and Ellipsis. Ms., City University of New York and University of California, Irvine.

Fiengo, Robert and Robert May. 1993. Indices and Identity, ms. City University of New York and University of California, Irvine.

Grice, H. Paul. 1968. Logic and Conversation. In Studies in the Way of Words. Cambridge: Harvard University Press.

Grimshaw, Joan. 1991 Extended Projection. Lecture notes, 1991 Linguistic Institute, University of California, Santa Cruz.

Grinder, John and Paul Postal. 1971. Missing Antecedents. Linguistic Inquiry 2:269-312.

Grodzinsky, Yosef and Tanya Reinhart. 1993. The Innateness of Binding and Coreference. Linguistic Inquiry 24.1:69-101.

Haïk, Isabelle. 1987. Bound VPs that Need to Be. Linguistics \& Philosophy 10:503-530.

Hankamer, Jorge and Ivan Sag. 1976. Deep and Surface Anaphora. Linguistic Inquiry, 7.3:391-426.

Hankamer, Jorge. 1978. On The Nontransformational Derivation of Some Null VP Anaphors. Linguistic Inquiry 9.1:66-74.

Heim, Irene. 1982. The Semantics of Definite and Indefinite Noun Phrases. Doctoral dissertation, University of Massachusetts, Amherst.

Heim, Irene. 1992. Anaphora and Semantic Interpretation: A Reinterpretation of Reinhart's Approach. Ms., MIT, Cambridge, Mass.

Higginbotham, James. 1983. Logical Form, Binding, and Nominals. Linguistic Inquiry 14.3:395-421.

Higginbotham, James. 1985. On Semantics. Linguistic Inquiry 16.4:547-593.

Hirschbühler, Paul. 1982. VP Deletion and Across the Board Quantifier Scope. IN Proceedings of NELS 12, 132-139, GSLA, University of Massachusetts, Amherst.

Huang, C.-T. James. 1993. Reconstruction and the Structure of VP: Some Theoretical Consequences. Linguistic Inquiry 24.1:103-138.

Jacobson, Pauline. 1992. ACD in a Variable Free Semantics. In Proceedings of SALT II. Kennedy, Christopher. 1993. A Constraint on Parallelism in Elliptical Constructions. Ms., University of California, Santa Cruz. 
Kitagawa, Yoshihisha. 1991. Copying Identity. Natural Language and Linguistic Theory 9:497536.

Kitagawa, Yoshihisha. 1986. Subjects in Japanese and English. Doctoral dissertation, University of Massachusetts, Amherst.

Koopman, Hilda and Dominique. Sportiche. 1988. Subjects. Ms. University of California, Los Angeles.

Kuroda, S.-Y. 1988. Whether We Agree or Not. Lingvisticae Investigationnes 12:1-47.

May, Robert. 1985. Logical Form. Cambridge: MIT Press.

Partee, Barbara. 1975. Montague Grammar and Transformational Grammar. Linguistic Inquiry 7.3.

Reinhart, Tanya. 1983. Coreference and Bound Anaphora: A Restatement of the Anaphora Questions. Linguistics \& Philosophy 6:47-88.

Reinhart, Tanya. 1987. Specifier and Operator Binding. In The Representation of (In)definiteness, ed. Eric Reuland and Alice Ter Meulen. Cambridge: MIT Press.

Rooth, Mats. 1981. A Comparison of Three Theories of Verb Phrase Ellipsis. In University of Massachusetts Occasional Papers in Linguistics 7:212-244.

Safir, Ken. 1986. Relative Clauses in a Theory of Binding and Levels. Linguistic Inquiry 17.4:663-689.

Sag, Ivan. 1976. Deletion and Logical Form. Doctoral dissertation, MIT, Cambridge, Mass.

Sag, Ivan and Jorge Hankamer. 1984. Toward a Theory of Anaphoric Processing. Linguistics \& Philosophy 7:325-345.

Wasow, Thomas. 1972. Anaphoric Relations in English. Doctoral dissertation, MIT, Cambridge, Mass.

Wescoat, Michael. 1989. Sloppy Readings with Embedded Antecedents. Ms., Stanford University.

Williams, Edwin. 1976. Discourse and Logical Form. Linguistic Inquiry 8.1:101-139.

Board of Studies in Linguistics

Stevenson College, UCSC

Santa Cruz, CA 95064

kennedy@ling.ucsc.edu 